\title{
THE CONVERGENCE WITH VANISHING VISCOSITY OF NONSTATIONARY NAVIER-STOKES FLOW TO IDEAL FLOW IN $R_{3}$
}

BY

\author{
H. S. G. SWANN
}

\begin{abstract}
It is shown here that a unique solution to the Navier-Stokes equations exists in $R_{3}$ for a small time interval independent of the viscosity and that the solutions for varying viscosities converge uniformly to a function that is a solution to the equations for ideal flow in $R_{3}$. The existence of the solutions is shown by transforming the Navier-Stokes equations to an equivalent system solvable by applying fixed point methods with estimates derived from using semigroup theory.
\end{abstract}

Introduction. We wish to find a solution, local in time, to the Cauchy problem for the Navier-Stokes equations for viscous incompressible flow in $R_{3}$ and show that the solutions of the Navier-Stokes equations for various viscosities converge, as the viscosity goes to zero, to a function that is a solution to the Euler equations for an ideal (inviscid) fluid.

The Navier-Stokes equations are

$$
\partial v / \partial t+(v \cdot \operatorname{grad}) v-v \Delta v=-\operatorname{grad} P+B, \quad \nabla \cdot v=0,
$$

with constraints

$$
\lim _{|x| \rightarrow \infty} v(x, t)=0 \quad \text { and } \quad v(x, 0)=C(x),
$$

where $x=\left(x_{1}, x_{2}, x_{3}\right)$ is a point in $R^{3} ; t$ is in some time interval $[0, T]$; the velociy $v(x, t)=\left(v_{1}(x, t), v_{2}(x, t), v_{3}(x, t)\right)$; the pressure is $P(x, t)$; the force is $B(x, t)$ $=\left(B_{1}(x, t), B_{2}(x, t), B_{3}(x, t)\right)$; and the constant $\nu>0$ is the viscosity (the coefficient of kinematic viscosity).

The Euler equations for ideal flow differ from the Navier-Stokes equations $\left(E^{\prime}\right)$ only in that the viscosity term $\nu \Delta v$ does not occur in the Euler equations.

Uniqueness and existence of a solution to the Navier-Stokes equations in $R^{3}$ has been shown for both bounded and unbounded domains: in both cases existence has been shown only for a sufficiently small time interval. The first results are those of C. W. Oseen [11] and Jean Leray [8]. The time interval where the solution is shown to exist must be small enough to satisfy a condition of form $T \leqq K \nu$, where $K$ is an appropriate constant and $\nu$ is the viscosity. Thus the length of the time interval

Received by the editors January 28, 1970 and, in revised form, June 25, 1970.

AMS 1968 subject classifications. Primary 7635, 7646; Secondary 3536, 4750.

Key words and phrases. Navier-Stokes equations, viscous flow, Euler equations, ideal flow, potential theory, semigroups of operators, Sobolev space, nonlinear partial differential equations. 
goes to zero (see [8, p. 223]) and will not allow us to consider the convergence of these solutions to the solution for ideal flow as the viscosity $v$ goes to zero. Later techniques of solution share this problem (see [1, pp. 142, 173]). Existence and uniqueness of a solution to the Euler equations for ideal flow, again for a sufficiently small time interval, was shown in $R^{3}$ by Leon Lichtenstein $[9$, p. 422] and on compact manifolds with boundary by Ebin and Marsden [15]. The existence, global in time, of "weak solutions" to the Navier-Stokes system was shown by Hopf [6], but satisfactory uniqueness results have not been found as yet. O. A. Ladyženskaja's recent book [1] provides an excellent survey of the various methods used for the solution of the Navier-Stokes equations and calls attention to the problem we consider in this paper $[1$, p. 6].

Convergence of viscous planar flow to ideal planar flow as the viscosity goes to zero was shown independently by McGrath [10] and Golovkin [5] with no restriction on the time interval of solution. Marsden has recently shown the existence for a short time (independent of viscosity) of viscous flow and its convergence to ideal flow on compact Riemannian manifolds without boundary using a technique suggested by V. Arnol'd [16]. We use an approach similar to that of McGrath and use techniques developed by Kato and Fujita [3], [4]. The result in this paper for $R^{3}$ differs from that of McGrath (for planar flow) in that we can demonstrate the existence of a unique classical solution to the Euler equations for ideal flow in $R^{3}$ by showing that the limit of solutions of the Navier-Stokes equations for various viscosities exists as the viscosity goes to zero, for a small but nontrivial time interval, and the limit function is a solution to the Euler equations for ideal flow. We call attention to the paper of Judovič [7] where he shows that the solution to the Euler equations for any domain in the plane is the limit of certain functions that are solutions of equations similar to the Navier-Stokes equations, but with a different form of boundary condition.

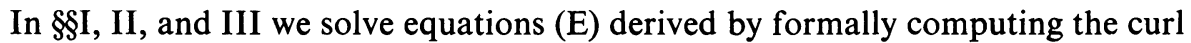
of the Navier-Stokes equations $\left(E^{\prime}\right)$ :

$$
\begin{aligned}
& \text { (a) } \partial w / \partial t+(v \cdot \operatorname{grad}) w-(w \cdot \operatorname{grad}) v-v \Delta w=\nabla \times B \equiv b . \\
& \text { (b) } w(x, 0)=\nabla \times C(x) \equiv a(x) . \\
& \text { (c) } \nabla \times v=w ; \nabla \cdot v=0 . \\
& \text { (d) } \lim _{|x| \rightarrow \infty} v(x, t)=0 .
\end{aligned}
$$

In solving the auxiliary problem (E) we use the following version of the Schauder fixed point theorem: Let $S$ be a closed convex subset of a Banach space $X$ and let $F$ be a continuous operator on $S$ such that $F(S)$ is contained in $S$ and $F(S)$ is a relatively compact subset of $X$. Then there is a "fixed point" $y$ in $S$, i.e. $F(y)=y$.

In $\S \mathrm{I}$ we show that for any $w$ in an appropriate class of functions there is a function $v=F_{1}(w)$ that solves $(\mathrm{E})(\mathrm{c})$ and $(\mathrm{d})$.

In $\S \mathrm{II}$, for $v=F_{1}(w)$, we show that there is a solution, denoted $F_{2}(v)$, to equations (E)(a) and (b) for any time interval. 
In §III it is shown that the function $F_{2}\left(F_{1}(\cdot)\right)$ maps a closed convex set of functions in a Banach space continuously into itself and satisfies the conditions of the Schauder fixed point theorem, provided we restrict ourselves to a sufficiently small time interval which is, however, independent of the viscosity. The fixed point $w$ is then shown to give a classical solution to $(\mathrm{E})$ and then $\left(\mathrm{E}^{\prime}\right)$.

In $§ I V$ we show that the solution $(w, v)$ to $(\mathrm{E})$ converges, with shrinking viscosity, to functions that give rise to a function that is a solution to the Euler equations for ideal flow.

The author is indebted to Professor Tosio Kato for his many helpful suggestions and comments during the preparation of this paper.

0. Preliminary results and definitions. The following notational conventions will be used:

$f, g$ and $h$ are scalar-valued functions over $R^{3}$ or $Q_{T}=R^{3} \times[0, T]$.

$B, C, a, b, p, q, u, v, w$ are vector-valued functions over $R^{3}$ or $Q_{T}$. For such functions, say $w$, we define $|w(x)|^{2}=\sum_{i=1}^{3}\left|w_{i}(x)\right|^{2}$.

Constants are denoted $K_{i}$ and do not depend on the viscosity $\nu$. The symbol $K$ denotes a constant used during a proof and $K$ may take different values during the same proof.

For $f \in L_{1}\left(R^{3}\right)$, the Fourier transform of $f$ is

$$
F(f)(x)=(2 \pi)^{-3 / 2} \int_{R^{3}} e^{-i x \cdot z} f(z) d z
$$

with the inverse Fourier transform of $f$ denoted $F^{-1}(f)$. By taking the limit-inmean, we can define the Fourier transform on $L_{2}\left(R^{3}\right)$, and, if $(,)_{L_{2}}$ denotes the inner product in Hilbert space $L_{2}\left(R^{3}\right)$, we have

$$
\|f\|_{L_{2}}^{2} \equiv(f, f)_{L_{2}}=(F(f), F(f))_{L_{2}}
$$

and

$$
\left(f_{1}, f_{2}\right)_{L_{2}}=\left(F\left(f_{1}\right), F\left(f_{2}\right)\right)_{L_{2}} .
$$

For $n \geqq 0$, the space $H^{n}$ is the completion of $C_{0}^{\infty}$ functions (infinitely differentiable functions with compact support in $R^{3}$ ) in the metric derived from norm

$$
\|f\|_{H^{n}}=\left\|F(f)(z)\left(1+|z|^{2}\right)^{n / 2}\right\|_{L_{2}}
$$

which, for $n$ an integer, is equivalent to the norm whose square is $\|f\|^{2}=$ $\sum_{|e| \leqq n}\left\|D_{x}^{e} f\right\|_{L_{2}}^{2}$ where $e=\left(e_{1}, e_{2}, e_{3}\right) ; e_{i}$ are integers $\geqq 0 ;|e|=e_{1}+e_{2}+e_{3}$ and $D_{x}^{e} f=\left(\partial / \partial x_{1}\right)^{e_{1}}\left(\partial / \partial x_{2}\right)^{e_{2}}\left(\partial / \partial x_{3}\right)^{e_{3}} f$.

$H_{\sigma}^{n}$ is the subspace of $H^{n}$ of all vector functions $u$ with $\nabla \cdot u=0$.

All explicit $D_{x}^{e} f$ are in $L_{2}$ and are understood as distribution derivatives. We note that $F\left(D_{x}^{e} f\right)(x)=(i x)^{e} F(f)(x)$, where $(i x)^{e}=\left(i x_{1}\right)^{e_{1}}\left(i x_{2}\right)^{e_{2}}\left(i x_{3}\right)^{e_{3}}$.

The following spaces will be used; $h$ may be a vector-valued or scalar-valued function. 
(i) $C_{T}=\left\{h(x, t) \mid h\right.$ is continuous and bounded in $\left.Q_{T}\right\}$ with norm $\|h\|_{C_{T}}$ $=\sup _{(x, t) \in Q_{T}}|h(x, t)|$.

(ii) $C_{T}^{\delta}=\left\{h(x, t) \mid D_{x}^{e} h(x, t) \in C_{T}\right.$ for all $e$ satisfying $|e| \leqq \delta$ and $D_{x}^{e} h$ is Höldercontinuous in $x$ with exponent $\delta-[\delta]$, uniformly for $(x, t) \in Q_{T}$ if $\left.|e|=[\delta]\right\}$ where [ $\left.\delta\right]$ is the largest integral part of $\delta \geqq 0$.

We use the norm whose square is

where

$$
\|h\|_{C_{T}^{\delta}}^{2}=\sum_{|e| \leqq[\delta]}\left\|D_{x}^{e} h\right\|_{C_{T}}^{2}+\sum_{|e|=[\delta]} H_{\delta}\left(D_{x}^{e} h\right)^{2}
$$

$$
H_{\delta}(f)=\sup _{x, x^{1} \in R^{3} ; t \in[0, T]} \frac{\left|f(x, t)-f\left(x^{1}, t\right)\right|}{\left|x-x^{1}\right|^{\delta-[\delta]}} .
$$

(iii) $C(T, H)=\{h(x, t) \mid h(, t) \in H$; the mapping $h$ : $[0, T] \rightarrow H$ is continuous $\}$ with norm $\|h\|_{C(T, H)}=\sup _{t \in[0, T]}\|h\|_{H}$ where $H$ is a Hilbert space, usually $H^{n}$ or $H_{\sigma}^{n}$. For notational convenience, we drop the $\sigma$ when subscripting the norm.

(iv) $C_{0}^{\delta}=\left\{h \in C_{T}^{\delta} \mid h\right.$ has compact support in $R^{3}$, uniformly in $\left.t \in[0, T]\right\}$.

$C_{0}^{\infty}$ is dense in $C\left(T, H^{n}\right)$. Where we consider $t$ only in an interval $[\varepsilon, T]$ with $\varepsilon>0$, we use analogous classes of functions $C_{[\varepsilon, T]}, C_{[\varepsilon, T]}^{\delta}$ and $C([\varepsilon, T], H)$. Where $t$ is omitted or fixed, we use similarly defined classes of functions $C, C^{\delta}$ and $C_{0}^{\delta}$.

$(,)_{H}$ denotes the inner product in a Hilbert space $H$. In any equation involving an inner product, the subscript $H$ will be used: subsequent inner products are assumed to be of the same kind unless the notation is changed.

LEMMA 0.1. If $f \in C\left(T, H^{n}\right)$ where $n$ is an integer, we can assume $f \in C_{T}^{n-2+\delta}$ for any $\delta$ with $0 \leqq \delta<\frac{1}{2}$ and there is a constant $K_{n, \delta}$ depending only on $n$ and $\delta$ such that

$$
\|f\|_{C_{T}^{n-2+\delta}} \leqq K_{n, \delta}\|f\|_{C\left(T, H^{n}\right)} .
$$

Proof. See [14, p. 221] for a proof for bounded domains. The proof for $R^{3}$ by use of Fourier transforms is somewhat easier.

LEMMA 0.2. Let $f_{1}$ and $f_{2}$ be scalar functions over $R^{3}$.

(i) If $f_{1} \in C$ and $f_{2} \in L_{2}$, then $f_{1} f_{2} \in L_{2}$ and

$$
\left\|f_{1} f_{2}\right\|_{L_{2}} \leqq\left\|f_{1}\right\|_{C}\left\|f_{2}\right\|_{L_{2}} \text {. }
$$

(ii) If $f_{1} \in H^{1}$ and $f_{2} \in H^{1}$, then $f_{1} f_{2} \in L_{2}$ and

$$
\left\|f_{1} f_{2}\right\|_{L_{2}} \leqq\left\|f_{1}\right\|_{H^{1}}\left\|f_{2}\right\|_{H^{1}}
$$

(iii) If $f_{1} \in C ; D_{x}^{e} f_{1} \in L_{2},|e|=1 ; \lim _{|x| \rightarrow \infty} f_{1}(x)=0$, then $f_{1} \in L_{6},\left\|f_{1}\right\|_{L_{6}} \leqq\left\|\nabla f_{1}\right\|_{L_{2}}$ where $\left\|\nabla f_{1}\right\|_{L_{2}}^{2}=\sum_{|e|=1}\left\|D_{x}^{e} f_{1}\right\|_{L_{2}}^{2}$, and if $f_{2} \in H^{1}$, then $f_{1} f_{2} \in L_{2}$ and

$$
\left\|f_{1} f_{2}\right\|_{L_{2}} \leqq\left\|\nabla f_{1}\right\|_{L_{2}}\left\|f_{2}\right\|_{H^{1}} .
$$

(iv) If $f \in L_{6}$ and $\nabla f \in H^{1}$, then $f$ can be taken as a locally Hölder-continuous function with exponent $\delta<\frac{1}{2}$. There is a constant $K_{1}(N, \delta)$, depending on $\delta$ and $N$, such that, in any ball $B(0, N)$,

$$
\|f\|_{C^{\delta}(B(0, N))} \leqq K_{1}(N, \delta)\left(\|f\|_{L_{6}}+\|\nabla f\|_{H^{1}}\right)
$$


where $\|f\|_{C^{\delta}(B(0, N))}$ is defined as in space $C^{\delta}$ over $B(0, N)$ instead of $R^{3} ; B(x, L)$ $=\left\{y \in R^{3}|| x-y \mid<L\right\}$.

Proof. (i) is immediate.

(ii) We can improve an inequality from [1, p. 12] to obtain, for all $f \in C_{0}^{\infty}$, $\|f\|_{L_{4}} \leqq\|f\|_{H^{1}}$. The result follows from a density argument applied to

$$
\left(\int f_{1}^{2} f_{2}^{2} d x\right)^{2} \leqq \int f_{1}^{4} d x \int f_{2}^{4} d x \leqq\left\|f_{1}\right\|_{H^{1}}^{4}\left\|f_{2}\right\|_{H^{1}}^{4}
$$

(iii) From [1, p. 12] we get, with some improvements, $\|f\|_{L_{6}} \leqq\|\nabla f\|_{L_{2}}$ if $f \in C_{0}^{\infty}$. Working in space $L_{2} \times L_{2} \times L_{2}$ of vector-valued functions whose components are in $L_{2}$ (denoted $L_{2}$ here), we let $D$ be the closure of $\left\{\nabla g \mid g \in C_{0}^{\infty}\right\}$ in this space. Since $\partial f_{1} / \partial x_{i} \in L_{2}, i=1,2,3$, we can find unique $w \in D, u \in L_{2} \ominus D$ such that $\nabla f_{1}=u+w$. Since $w \in D$, there is a sequence $\left\{g_{i}\right\} \subset C_{0}^{\infty}$ such that $\nabla g_{i} \rightarrow w$ in $L_{2}$ as $i \rightarrow \infty$. The inequality above holds for $g_{i}$, so there is some $g \in L_{6}$ such that $g_{i} \rightarrow g$ and $w \in D$ is the distribution gradient of $g$. Thus, for $f \in C_{0}^{\infty}$,

$$
0=(u, \nabla f)_{L_{2}}=\left(\nabla f_{1}-w, \nabla f\right)=\left(f_{1}-g, \Delta f\right) .
$$

So $f_{1}-g$ must be harmonic in $R^{3}$; but $f_{1} \in C ; \lim _{|x| \rightarrow \infty} f_{1}(x)=0$ and $g \in L_{6}$, which can only occur if $f_{1}-g=0$, by standard results concerning harmonic functions. Thus

$$
\left\|f_{1}\right\|_{L_{6}}=\|g\|_{L_{6}} \leqq\|\nabla g\|_{L_{2}}=\|w\|_{L_{2}} \leqq\left\|\nabla f_{1}\right\|_{L_{2}} .
$$

Now, using Hölder's inequality, we compute

$$
\begin{aligned}
\left\|f_{1} f_{2}\right\|_{L_{2}}^{2} & \leqq\left(\int f_{1}^{6} d x\right)^{1 / 3}\left(\int\left(f_{2}^{2}\right)^{3 / 2} d x\right)^{2 / 3} \\
& \leqq\left\|\nabla f_{1}\right\|_{L_{2}}^{2}\left\|f_{2}\right\|_{L_{2}}^{2 / 3}\left\|f_{2}\right\|_{L_{4}}^{4 / 3} \leqq\left\|\nabla f_{1}\right\|_{L_{2}}^{2}\left\|f_{2}\right\|_{H^{1}}^{2}
\end{aligned}
$$

using the inequality $\left\|f_{2}\right\|_{L_{4}} \leqq\left\|f_{2}\right\|_{H^{1}}$.

(iv) This result can easily be obtained by multiplying $f$ by a function $g \in C_{0}^{\infty}$ that equals 1 on $B(0, N)$, using Lemma 0.1 on $f g$, and the result

$$
\int_{B(0, N)} f^{2} d x \leqq K\|f\|_{L_{6}}^{2} \quad(K \text { depends on } N) .
$$

LEMMA 0.3. If a set of functions $S \subset L_{2}$ has a uniform Hölder constant $M$ and exponent $\delta$ and if, for any $\varepsilon>0$, there is an $N_{\varepsilon}$ such that

$$
\int_{R^{3}-B\left(0, N_{\varepsilon}\right)}|f|^{2} d x<\varepsilon \text { for all } f \in S,
$$

then for any $\varepsilon^{1}$, there is an $N_{\varepsilon^{1}}$ such that $|x|>N_{\varepsilon^{1}}$ implies that $|f(x)|<\varepsilon^{1}$ for all $f \in S$.

Proof. Suppose that $S$ has the properties postulated in the lemma and there is some $f_{1} \in S$ and $x_{1} \in R^{3}-B(0, N+1)$ with

$$
\left|f_{1}\left(x_{1}\right)\right| \geqq L \equiv\left(6 \varepsilon(3+\delta)(3+2 \delta)\left(8 \pi \delta^{2}\right)^{-1} M^{3 / \delta}\right)^{\delta /(2 \delta+3)} \text {. }
$$


Then $\left|f_{1}(x)\right| \geqq L-M\left|x-x_{1}\right|^{\delta}$ and

$$
\begin{aligned}
\int_{R^{3}-B(O, N)}\left|f_{1}\right|^{2} d x & \geqq \int_{B\left(x_{1},(L / M)^{1 / \delta}\right)}\left(L-M\left|x-x_{1}\right|^{\delta}\right)^{2} d x \\
& =4 \pi \int_{0}^{(L / M)^{1 / \delta}}\left(L^{2}-2 L M r^{\delta}+M^{2} r^{2 \delta}\right) r^{2} d r=2 \varepsilon,
\end{aligned}
$$

which contradicts the assumption that $f_{1} \in S$. Hence $|f(x)| \leqq L$, all $f \in S$, all $x \in R^{3}$ $-B(0, N+1)$ and the conclusion is immediate from this.

I. In $\S$ I we show that if $w \in H_{\sigma}^{2}$ then there is a unique $v \in C^{1+\delta} \cap L_{6}$ such that $\nabla \times v=w$ and $\lim _{|x| \rightarrow \infty}|v(x)|=0$. We use potential theory to construct $v$.

LEMMA 1.1. For any scalar function $f \in C^{\delta} \cap L_{2}$ we can define a linear operator

$$
G(f)(x)=\int_{R^{3}}\left(\frac{1}{|x-y|}-\frac{1}{|y|}\right) f(y) d y .
$$

$G(f)$ is twice continuously differentiable, $\Delta G(f)=-4 \pi f$ and $\|\nabla G(f)\|_{C} \leqq$ $4 \pi\left(\|f\|_{C}+\|f\|_{L_{2}}\right)$.

Proof. For any $x \in R^{3}$, we can choose $z \in R^{3}$ such that $|z-x|<1$. Then

$$
\begin{aligned}
G(f)(x) & =\int_{R^{3}}\left(|x-y|^{-1}-|y|^{-1}\right) f(y) d y \\
& =\int_{B(z, 2)}+\int_{B(0,1)}+\int_{R^{3}-B(z, 2)-B(0,1)} \\
& \equiv I_{1}+I_{2}+I_{3} .
\end{aligned}
$$

By potential theory arguments (see [2, p. 249]) $I_{1}$ and $I_{2}$ exist and are twice continuously differentiable;

$$
\frac{\partial I}{\partial x_{j}}=\int_{B} \frac{\partial}{\partial x_{j}} \frac{1}{|x-y|} f(y) d y \text { for both } I_{1} \text { and } I_{2} \text {. }
$$

By Schwarz inequality and ||$y|-| x-y|| \leqq|x|$,

$$
\begin{aligned}
\left|I_{3}\right|^{2} & \leqq\|f\|_{L_{2}}^{2} \int_{R^{3}-B(z, 2)-B(0,1)}\left(\frac{|y|-|x-y|}{|y||x-y|}\right)^{2} d y \\
& \leqq\|f\|_{L_{2}}^{2}|x|^{2} \int_{R^{3}-B-B}|y|^{-2}|x-y|^{-2} d y \leqq K\|f\|_{L_{2}}^{2}|x|^{2} .
\end{aligned}
$$

This is sufficient for $I_{3}$ to exist; showing that it is sufficiently differentiable uses an argument similar to the following reasoning concerning the desired inequality:

$$
\begin{aligned}
|\nabla G(f)(x)| & \leqq \int_{R^{3}}\left|\nabla\left(|x-y|^{-1}\right)\right||f(y)| d y \\
& =\int_{R^{3}}|x-y|^{-2}|f(y)| d y \\
& \leqq \int_{R^{3}-B(x, 1)}|x-y|^{-2}|f(y)| d y+\|f\|_{C} \cdot 4 \pi \int_{0}^{1} d r \\
. & \leqq 4 \pi\left(\|f\|_{L_{2}}+\|f\|_{C}\right) .
\end{aligned}
$$


Finally, from potential theory (see [2, p. 249]), $\Delta G(f)=-4 \pi f$.

Lemma 1.2. Let $w \in C^{\delta} \cap H_{\sigma}^{0}$. Then if $G(w)=\left(G\left(w_{1}\right), G\left(w_{2}\right), G\left(w_{3}\right)\right)($ see Lemma 1.1),

(i) $\lim _{|x| \rightarrow \infty}\left|\partial G(w) / \partial x_{i}\right|=0$, and

(ii) $\nabla \cdot G(w)=0$.

Proof. (i) The absolute value of a component of $\partial G(w)(x) / \partial x_{i}$ is

$$
\left|\int_{R^{3}}\left(\left(x_{i}-y_{i}\right)|x-y|^{-3}\right) w_{j}(y) d y\right| \leqq \int_{R^{3}}|x-y|^{-2}\left|w_{j}(y)\right| d y .
$$

Hence it suffices to show that $f \in C^{\delta} \cap L_{2}$ implies

$$
\lim _{|x| \rightarrow \infty} \int_{R^{3}} \frac{1}{|x-y|^{2}} f(y) d y=0 .
$$

Using Lemma 0.3 with $S=\{f\}$, for any $\varepsilon>0$ we can find $N$ such that $\int_{R^{3}-B(0, N)}|f|^{2} d x<\varepsilon^{2}$ and $|f(x)|<\varepsilon$ if $x \in R^{3}-B(0, N)$. Suppose $|x|>N+1$. Write

$$
\begin{gathered}
\int_{R^{3}}|x-y|^{-2} f(y) d y=\int_{R^{3}-B(0, N)}+\int_{B(0, N)} \equiv I_{1}+I_{2}, \\
\left.\left|I_{1}\right| \leqq \int_{R^{3}-B(0, N)-B(x, 1)}+\int_{B(x, 1)}|x-y|^{-4} d y\right)^{1 / 2}\left(\int_{R^{3}-B(0, N)} f^{2} d y\right)^{1 / 2}+K \sup _{y \in B(x, 1)}|f(y)| \leqq K \varepsilon \\
\leqq\left(\int_{R^{3}-B(x, 1)} \mid\right. \\
\left|I_{2}\right|^{2} \leqq\|f\|_{L_{2}}^{2} \int_{B(0, N)}|x-y|^{-4} d y \\
\leqq\|f\|_{L_{2}}^{2}(|x|-N)^{-1 / 2} \int_{R^{3}-B(x, 1)}|x-y|^{-7 / 2} d y \\
\leqq K(|x|-N)^{-1 / 2}\|f\|_{L_{2}}^{2} \rightarrow 0 \text { as }|x| \rightarrow \infty .
\end{gathered}
$$

These suffice to establish (i).

(ii) The result $\nabla \cdot G(w)=0$ follows from a conventional procedure involving approximating $|x-y|^{-1}$ in $B(x, 2 N)-B(x, \delta / 2)$ by a $C_{0}^{\infty}$ function $f_{N, \delta}(|x-y|)$ equal to $|x-y|^{-1}$ in $B(x, N)-B(x, \delta)$. Then, for $f_{N, \delta}, \nabla \cdot w=0$ implies

$$
\sum \frac{\partial}{\partial x_{i}} \int f_{N, \delta}(|x-y|) w_{i}(y) d y=-\left(\nabla f_{N, \delta}(|x-y|), w\right)_{L_{2}}=0 .
$$

THEOREM 1.1. If $w \in H_{\sigma}^{n}(n \geqq 2)$ we can define a linear map $F_{1}$ :

$$
F_{1}(w)(x)=(4 \pi)^{-1} \int_{R^{3}}|x-y|^{-3}(x-y) \times w(y) d y=(4 \pi)^{-1} \nabla \times G(w)
$$

with the following properties:

(a) $F_{1}(w) \in C^{1+\delta} \cap L_{6}$ for any $\delta<\frac{1}{2}$ and $\partial F_{1}(w) / \partial x \in H^{n}$,

(b) $\nabla \times F_{1}(w)=w$, 
(c) $\nabla \cdot F_{1}(w)=0$,

(d) $\lim _{|x| \rightarrow \infty} F_{1}(w)(x)=0$.

$F_{1}(w)$ is the unique vector with properties (a) through (d).

For a vector-valued function $u=\left(u_{1}, u_{2}, u_{3}\right)$, let $u_{i, x_{j}}=\partial u_{i} / \partial x_{j}$, and $u_{, x}$ be the array $\left(u_{i, x_{j}}\right)$ and

$$
\left\|u_{, x}\right\|^{2}=\sum_{i, j=1,2,3}\left\|u_{i, x_{j}}\right\|^{2} .
$$

The following inequalities hold:

(i) $\left\|F_{1}(w)\right\|_{L_{6}} \leqq K_{2}\|w\|_{L_{2}}$,

(ii) $\left\|F_{1}(w)\right\|_{C} \leqq K_{3}\|w\|_{H^{2}}$,

(iii) $\left\|\left(F_{1}(w)\right), x\right\|_{H^{m}} \leqq\|w\|_{H^{m}}, m=0,1,2, \ldots, n$.

Proof. Since $w \in H^{n}$, where $n \geqq 2$, Lemma 0.1 shows that $w \in C^{\delta}$ for any $\delta<\frac{1}{2}$. Hence Lemmas 1.1 and 1.2 are valid and $F_{1}(w)$ exists. Using these lemmas,

$$
\nabla \times F_{1}(w)=\nabla \times\left((4 \pi)^{-1} \nabla \times G(w)\right)=(4 \pi)^{-1}(-\Delta G(w)+\nabla(\nabla \cdot G(w)))=w,
$$

where $\Delta G(w)=\left(\Delta G\left(w_{1}\right), \Delta G\left(w_{2}\right), \Delta G\left(w_{3}\right)\right) . \lim _{|x| \rightarrow \infty} F_{1}(w)=0$ by Lemma $1.2(\mathrm{i})$.

To establish uniqueness: If $v_{1}$ and $v_{2}$ both have properties (a) through (d), then $\nabla \times\left(v_{1}-v_{2}\right)=0$ and $\nabla \cdot\left(v_{1}-v_{2}\right)=0$, so there is a potential function $f$ with $v_{1}-v_{2}$ $=\nabla f$ and $0=\nabla \cdot\left(v_{1}-v_{2}\right)=\Delta f$, i.e. $f$ is harmonic. But $v_{1}$ and $v_{2}$ are small near $\infty$, so $f$, harmonic, can only be constant; hence $v_{1}=v_{2}$.

$F_{1}$ is clearly linear; inequality (ii) follows easily from the inequality of Lemma 1.1 and Lemma 0.1 .

To establish the remaining results, we first show that, if $v=F_{1}(w)$, then

$$
v_{, x_{i}}=-F^{-1}\left(|z|^{-2} z_{i}(z \times F(w)(z))\right),
$$

where $F$ is the Fourier transform. Let $u=-(4 \pi)^{-1} G(w)$. Then $\Delta u=w$ and $\nabla \times u$ $=-v$. Denote

$$
-F^{-1}\left(|z|^{-2} z_{i}(z \times F(w)(z))\right)
$$

by $p_{i}$; note that $p_{i} \in H^{n}$. Then if $q \in C_{0}^{\infty}$,

$$
\begin{aligned}
\left(p_{i}, \Delta q\right)_{L_{2}} & =\left(-|z|^{-2} z_{i}(z \times F(w)(z)),|z|^{2} F(q)(z)\right) \\
& =-\left(F(w)(z), z_{i}(z \times F(q)(z))\right)=\left(F(\Delta u), F\left((\nabla \times q)_{, x_{i}}\right)\right) \\
& =\left(\Delta u,(\nabla \times q)_{, x_{i}}\right)=\left(u, \Delta(\nabla \times q)_{, x_{i}}\right)=-\left((\nabla \times u)_{, x_{i}}, \Delta q\right) \\
& =\left(v_{, x_{i}}, \Delta q\right) .
\end{aligned}
$$

Hence $v, x_{i}-p_{i}$ is harmonic; $v, x_{i}$ and $p_{i}$ are both continuous and bounded by Lemmas 1.1 and 0.1. So $v, x_{i}=p_{i}+$ constant. Now $p_{i} \in H^{2}$, so Lemma 0.1 implies that $p_{i} \in C^{\delta} \cap L_{2}$ and Lemma 0.3 implies that $p_{i}$ is uniformly small outside a sufficiently large ball in $R^{3}$. Since $v$ is also small uniformly for $x$ large, the mean- 
value theorem implies that for at least some large $x_{0}, v,{ }_{, x}\left(x_{0}\right)$ is also small; hence the "constant" must be 0 and $v, x_{i}=p_{i}$. Thus (iii) follows:

$$
\begin{aligned}
\|v, x\|_{H^{n}}^{2} & =\sum_{i}\left\|F\left(v_{, x_{i}}\right)(z)\left(1+|z|^{2}\right)^{n / 2}\right\|_{L_{2}}^{2} \\
& =\left\||z|^{-2}(|z||z \times F(w)(z)|)\left(1+|z|^{2}\right)^{n / 2}\right\|_{L_{2}}^{2} \\
& \leqq\left\|F(w)\left(1+|z|^{2}\right)^{n / 2}\right\|_{L_{2}}^{2}=\|w\|_{H^{n}}^{2} .
\end{aligned}
$$

Inequality (i) now can easily be derived using Lemma 0.2 (iii); $v=F_{1}(w) \in C^{1+\delta}$ since $v, x \in H^{2}$ and Lemma 0.1 holds.

II. In $§ I$, for fixed $v$, we wish to find a solution to

(a) $\quad \partial w / \partial t+(v \cdot \operatorname{grad}) w-(w \cdot \operatorname{grad}) v-\nu \Delta w=b$,

(b) $\quad w(x, 0)=a(x)$.

Equations (E)(a) and (b) provide an example of more general parabolic equations of form

(a) $d w / d t+(P(t)+A) w=b$,

(b) $w(0)=a$,

where $A$ is selfadjoint and independent of time and $P(t)$ is a time dependent linear "perturbation of lower order." The following theorem provides a solution to equations of form $(\mathrm{Q}) \cdot(D(\cdot)$ denotes the domain of operator.)

THEOREM 2.1. Let $A$ be a selfadjoint operator in a Hilbert space $H$ and suppose that $A \geqq d>0$. Let $P(t)$ be a time-dependent linear operator with $D(P(t)) \supset D\left(A^{\delta}\right)$ for some $\delta \geqq 0$ and all $t \in[0, T]$. Suppose that if $w \in D\left(A^{\delta}\right)$, then $P(t) w \in C(T, H)$ and

$$
\|P(t) w\|_{C(T, H)} \leqq K\left\|A^{\delta} w\right\|_{H} \quad(K \text { is some constant }) .
$$

(I) If $a \in D\left(A^{\delta}\right)$ and $b \in C(T, H)$, then there is a generalized solution $w(t) \in C(T, H)$ to (Q) which satisfies

(a) $w(t)=e^{-t A} a+\int_{0}^{t} e^{-(t-s) A}(-P(s) w(s)+b(s)) d s$

$$
\text { where } e^{-t A} \text { is the semigroup generated by }-A \text {; and }
$$

(b) $w(t) \in D\left(A^{\delta}\right), t \in[0, T], A^{\delta} w(t) \in C(T, H)$ and

$$
\lim _{t \downarrow 0}\left\|A^{\delta}(w(t)-a)\right\|_{H}=0 \text {. }
$$

(II) If $a \in D\left(A^{\delta+\mu}\right)$ for some $\mu>0, b$ is Hölder continuous in $t$ as $a C(T, H)$ function, and $P(t)$ also satisfies

$$
\left\|P\left(t_{1}\right) u-P\left(t_{2}\right) u\right\|_{H} \leqq K^{\prime}\left|t_{1}-t_{2}\right|^{\mu}\left\|A^{\delta} u\right\|_{H}
$$

for some constants, $K^{\prime}, \mu>0$, and all $u \in D\left(A^{\delta}\right)$, then $w(t)$ is also a solution of $(\mathrm{Q})$ in the sense that $d w / d t$ exists in $C([\varepsilon, T], H)$ for any $\varepsilon>0 ; w(t) \in D(A)$ for $t>0$ and (Q) is satisfied in $H$ for all $t>0$. 
The following lemma is needed for the proof of Theorem 2.1; the dependence of estimates on the positive constant $\nu$ is crucial for later results.

LeMmA 2.1. If $A$ is a positive selfadjoint operator in Hilbert space $H, A \geqq d>0$, then $-A$ is the generator of a contraction semigroup $e^{-t A}$ and

(i) $e^{-t A} e^{-s A}=e^{-(t+s) A}, t, s>0 ; A e^{-t A} \supset e^{-t A} A ;\left\|e^{-t A}\right\| \leqq 1$.

(ii) $e^{-t A} \rightarrow I$ strongly as $t \rightarrow 0$.

(iii) $e^{-t A}$ maps $H$ into $D(A)(t>0)$.

(iv) $d\left(e^{-t A}\right) / d t=-A e^{-t A}$ exists $(t>0)$.

(v) $e^{-t A} w \in C(T, H)$ for all $w \in H$.

(vi) If $a \in H$ and $b(s)$ is Hölder-continuous as a $C(T, H)$ function, then

$$
w(t)=e^{-t A} a+\int_{0}^{t} e^{-(t-s) A} b(s) d s
$$

solves

(a) $d w / d t+A w=b$,

(b) $w(0)=a$,

in the sense that $w(t) \in D(A), t>0$ and $d w / d t$ and $A w(t)$ exist and (a) is true in $C([\varepsilon, T], H)$ for $\varepsilon>0$ and $\lim _{t \downarrow 0}\|w(t)-a\|_{H}=0$.

(vii) If $0 \leqq \delta<1,\left\|A^{\delta} u\right\|_{H} \geqq d^{\delta}\|u\|_{H}$ for all $u \in D\left(A^{\delta}\right)$.

(viii) $\left\|A^{\delta} e^{-t A}\right\| \leqq t^{-\delta}(\delta / e)^{\delta}$.

(ix) $\left\|\left(e^{-h A}-1\right) A^{-\delta}\right\| \leqq h^{\delta}((1-\delta) / e)^{1-\delta} \delta^{-1}$.

(x) For any $u \in C(T, H)$,

$$
\left\|\int_{0}^{t} A^{\delta} e^{-(t-s) v A} u(s) d s\right\|_{H} \leqq t^{1-\delta} \nu^{-\delta}(\delta / e)^{\delta}(1-\delta)^{-1}\|u\|_{C(T, H)} .
$$

(xi) If $w(t)=\int_{0}^{t} e^{-(t-s) v A} u(s) d s$, where $u(s) \in C(T, H)$, then, for any $0 \leqq \delta<1$ and $0<\mu<1-\delta, A^{\delta} w(t) \in C(T, H)$ and

$$
\left\|A^{\delta}(w(t+h)-w(t))\right\|_{H} \leqq h^{\mu} \nu^{-\delta}\left((t / \mu)^{1-(\delta+\mu)}+(1-\delta)^{-1} h^{1-(\delta+\mu)}\right)\|u\|_{C(t+h, H)} .
$$

Proof. The results (i) through (viii) are well known; for example, see [12, p. $231 \mathrm{ff}$.] and [13].

(ix) $\left(e^{-h A}-1\right)=\int_{0}^{h}(d / d t) e^{-t A} d t=\int_{0}^{h}-A e^{-t A} d t$ so

$$
\left(e^{-h A}-1\right) A^{-\delta}=\int_{0}^{h}-A^{1-\delta} e^{-t A} d t
$$

and the result follows from integrating inequality (viii).

(x) can be easily shown by integrating (viii) directly.

(xi) $w(t+h)-w(t)=\left(e^{-h v A}-1\right) \int_{0}^{t} e^{-(t-s) v A} u(s) d s+\int_{t}^{t+h} e^{-(t+h-s) v A} u(s) d s$ so

$$
\begin{aligned}
& \left\|A^{\delta}(w(t+h)-w(t))\right\|_{H} \\
& \quad \leqq\left\|\left(e^{-h v A}-1\right) A^{-\mu}\right\|\left\|\int_{0}^{t} A^{\delta+\mu} e^{-(t-s) v A} u(s) d s\right\|+\left\|\int_{t}^{t+h} A^{\delta} e^{-(t+h-s) v A} u(s) d s\right\| .
\end{aligned}
$$

The result follows easily from the previous estimates. 


\section{Proof of Theorem 2.1.}

Part I. We solve the integral equation $\left(Q^{1}\right)(a)$ by an approximation technique, with

and

$$
u_{0}(t)=e^{-t A} a+\int_{0}^{t} e^{-(t-s) A} b(s) d s
$$

$$
u_{n}(t)=-\int_{0}^{t} e^{-(t-s) A} P(s) u_{n-1}(s) d s .
$$

Now $u_{0}(t)$ exists in $C(T, H)$ by Lemma 2.1(xi) (for $\delta=0$ ) which also allows us to assert that

$$
A^{\delta} u_{0}(t)=e^{-t A} A^{\delta} a+\int_{0}^{t} A^{\delta} e^{-(t-s) A} b(s) d s
$$

exists in $C(T, H)$ and so, by the assumptions of the theorem, $u_{0}(t) \in D(P(t))$. Assume that $u_{n-1}(t)$ and $A^{\delta} u_{n-1}(t)$ exist in $C(T, H)$ so that $u_{n-1}(t) \in D(P(t))$. We note that for any $w(t) \in D\left(A^{\delta}\right), t \in[0, T]$,

$$
\left\|P\left(t_{1}\right) w\left(t_{1}\right)-P\left(t_{2}\right) w\left(t_{2}\right)\right\|_{H} \leqq\left\|\left\{P\left(t_{1}\right)-P\left(t_{2}\right)\right\} w\left(t_{1}\right)\right\|_{H}+K\left\|A^{\delta}\left(w\left(t_{1}\right)-w\left(t_{2}\right)\right)\right\|_{H}
$$

from the properties assumed of $P$. Hence $P(t) u_{n-1}(t) \in C(T, H)$. Then, by Lemma 2.1(xi), $u_{n}(t)$ and $A^{\delta} u_{n}(t)$ exist in $C(T, H)$. The inequalities

and

$$
\left\|A^{\delta} u_{0}\right\|_{C(t, H)} \leqq\left\|A^{\delta} a\right\|_{H}+t^{1-\delta}(1-\delta)^{-1}\|b\|_{C(t, H)}
$$

$$
\begin{aligned}
\left\|A^{\delta} u_{n}\right\|_{C(t, H)} & \leqq t^{1-\delta}(1-\delta)^{-1}\left\|P u_{n-1}\right\|_{C(t, H)} \\
& \leqq t^{1-\delta}(1-\delta)^{-1} K\left\|A^{\delta} u_{n-1}\right\|_{C(t, H)}
\end{aligned}
$$

follow from Lemma 2.1(x) and the restrictions on $P$. Thus

$$
\sum_{i=0}^{n}\left\|A^{\delta} u_{i}\right\|_{C(t, H)} \leqq\left(\left\|A^{\delta} a\right\|_{H}+t^{1-\delta}(1-\delta)^{-1}\|b\|_{C(t, H)}\right) \sum_{i=0}^{n}\left(t^{1-\delta}(1-\delta)^{-1} K\right)^{i}
$$

which converges if $t$ satisfies $t^{1-\delta}(1-\delta)^{-1} K<1$. Note that this restriction on $t$ is independent of the initial data and $b(t)$. If $T_{0}$ satisfies this restriction, then the series $\left(A^{\delta} w\right)_{n} \equiv \sum_{i=0}^{n} A^{\delta} u_{i}$ converges in $C\left(T_{0}, H\right)$ and since $d^{\delta}\left\|u_{i}\right\|_{H} \leqq\left\|A^{\delta} u_{i}\right\|_{H}, w_{n}$ $\equiv \sum_{i=0}^{n} u_{i}$ will converge also to some function $w \in C\left(T_{0}, H\right)$. Since $A^{\delta}$ is closed, $\lim _{n \rightarrow \infty}\left(A^{\delta} w\right)_{n}=A^{\delta} w$ and hence $w \in D(P(t))$, and

$$
\begin{aligned}
w(t) & =u_{0}(t)+\lim _{n \rightarrow \infty} \sum_{i=1}^{n} u_{i}(t) \\
& =u_{0}(t)-\lim _{n \rightarrow \infty} \int_{0}^{t} e^{-(t-s) A} P(s) \sum_{i=0}^{n} u_{i}(s) d s \\
& =u_{0}(t)-\int_{0}^{t} e^{-(t-s) A} P(s) w(s) d s .
\end{aligned}
$$

Now $w\left(T_{0}\right) \in D\left(A^{\delta}\right)$; hence this process can be continued to another $t$-interval $\left[T_{0}, 2 T_{0}\right]$ of the same length etc. since the requirement for convergence of the 
approximation is independent of the initial data. So $w(t)$ satisfying the integral equation exists for any interval $[0, T]$ where $b(t)$ and $P(t)$ are defined. We note that

$$
A^{\delta} w(t)-A^{\delta} a=\left(e^{-t A}-1\right) A^{\delta} a+\int_{0}^{t} A^{\delta} e^{-(t-s) A}(-P w+b) d s
$$

and the proper convergence to the initial value follows from Lemma 2.1(ii) and (x).

Part II. To show the second part of the result, we need only show that $P(s) w(s)$ is Hölder-continuous in $s$ by Lemma 2.1(vi). Inequality (2.1) and the additional assumptions on $P$ reduce this to showing that $A^{\delta} w(s)$ is Hölder-continuous. First

$$
e^{-(s+h) A} A^{\delta} a-e^{-s A} A^{\delta} a=\left(\left(e^{-h A}-1\right) A^{-\mu}\right)\left(e^{-s A} A^{\delta+\mu} a\right)
$$

and inequalities (viii) and (ix) of Lemma 2.1 show that $A^{\delta} e^{-s A} a$ is Hölder-continuous if $a \in D\left(A^{\delta+\mu}\right)$, as is assumed.

Then Lemma 2.1(xi) establishes that

$$
A^{\delta}\left(\int_{0}^{t} e^{-(t-s) A}(-P(s) w(s)+b(s)) d s\right)
$$

is Hölder-continuous in $t$ also.

Let $A^{\prime}$ be the closure of $(1-\Delta)$ on Hilbert space $H^{2}$; then $D\left(A^{\prime}\right)=H^{4}$. Now it is clear that for sufficiently smooth $q, \nabla \cdot\left(A^{\prime} q\right)=A^{\prime}(\nabla \cdot q)$ where $A^{\prime}$ is regarded as an operator on both scalar- and vector-valued functions. Hence $A=\left(A^{\prime}\right.$ restricted to $H_{\sigma}^{2}$ ) is an operator in this subspace with domain $H_{\sigma}^{4}$, and $\nu A$ is the selfadjoint operator in Hilbert space $H_{\sigma}^{2}$ for use with Theorem 2.1 to show the existence of solutions of equations (E).

Formally define

$$
P_{v, v}(t) w=(v \cdot \operatorname{grad}) w-(w \cdot \operatorname{grad}) v-\nu w .
$$

Recalling that $\nabla \cdot v=0$, for smooth $u$ with $\nabla \cdot u=0$,

$$
\begin{aligned}
\nabla \cdot P_{v, v} u & =\nabla \cdot((v \cdot \operatorname{grad}) u)-\nabla \cdot((u \cdot \operatorname{grad}) v)-\nu \nabla \cdot u \\
& =\frac{\partial v_{j}}{\partial x_{i}} \frac{\partial}{\partial x_{j}} u^{i}+(v \cdot \operatorname{grad})(\nabla \cdot u)-\frac{\partial}{\partial x_{j}} u^{i} \frac{\partial v_{j}}{\partial x_{i}}-(u \cdot \operatorname{grad})(\nabla \cdot v)+0=0 .
\end{aligned}
$$

Thus we can define $P_{v, v}(t)$ in $H_{\sigma}^{2}$. For use in Theorem 2.1 we need the following estimates.

Note that, from Lemma 0.1 , there is a constant $K_{7}$ such that, for suitable $q$, $\|q, x\|_{C} \leqq K_{7}\|q, x\|_{H^{2}}$ and $\|q\|_{C} \leqq K_{7}\|q\|_{H^{2}}$.

LEMMA 2.2. If $v \in C_{T}, \nabla \cdot v=0, \lim _{|x| \rightarrow \infty} v(x, t)=0$ uniformly in $t \in[0, T]$ and $v, x \in C\left(T, H^{2}\right)$, then, for suitable constants $K_{4}, K_{5}$ and $K_{6}$, if $w \in D\left(A^{1 / 2}\right)=H_{\sigma}^{3}$,

(i) $\|(v \cdot \operatorname{grad}) w\|_{C\left(T, H^{2}\right)} \leqq K_{4}\left(\|v, x\|_{C\left(T, H^{2}\right)}+\|v\|_{C_{T}}\right)\left\|A^{1 / 2} w\right\|_{H_{\sigma}^{2}}$,

(ii) $\|(w \cdot \operatorname{grad}) v\|_{C\left(T, H^{2}\right)} \leqq K_{5}\|v, x\|_{C\left(T, H^{2}\right)}\|w\|_{H_{\sigma}^{2}}$,

(iii) $\|(v \cdot \operatorname{grad}) w\|_{C\left(T, H^{1}\right)} \leqq K_{6}\left(\|v, x\|_{C\left(T, H^{2}\right)}+\|v\|_{C_{T}}\right)\|w\|_{H_{\sigma}^{2}}$. 
Proof. For appropriate $p$ and $q$,

and

$$
\begin{aligned}
\|(p \cdot \operatorname{grad}) q\|_{H_{2}} & =\|(1-\Delta)((p \cdot \operatorname{grad}) q)\|_{L_{2}} \\
& \leqq\|(p \cdot \operatorname{grad}) q\|_{L_{2}}+\|\Delta((p \cdot \operatorname{grad}) q)\|_{L_{2}}
\end{aligned}
$$

$$
\Delta((p \cdot \operatorname{grad}) q)=(\Delta p \cdot \operatorname{grad}) q+2 \sum_{i}\left(p_{, x_{i}} \cdot \operatorname{grad}\right) q_{, x_{i}}+(p \cdot \operatorname{grad}) \Delta q .
$$

\section{Proof of (i).}

$$
\begin{aligned}
& \|(p \cdot \operatorname{grad}) q\|_{L_{2}} \leqq\|p\|_{C}\|q,\|_{L_{2}}, \\
& \|\Delta((p \cdot \operatorname{grad}) q)\|_{L_{2}} \leqq K\left\|p_{, x}\right\|_{H^{2}}\left\|q{ }_{, x}\right\|_{H^{1}}+K\left\|p_{, x}\right\|_{C}\left\|q{ }_{, x}\right\|_{H^{1}}+\|p\|_{C}\left\|\Delta q_{, x}\right\|_{L_{2}} \text {, } \\
& \left\|q_{, x}\right\|_{H_{2}}^{2}=\sum_{i, j}\left\|q_{i, x}\right\|_{H_{2}}^{2} \leqq \int\left(1+|z|^{2}\right)^{3}|F(q)|^{2} d z=\left\|A^{\prime 1 / 2} q\right\|_{H^{2}}^{2} .
\end{aligned}
$$

Lemma 0.2 gives $\left\|p_{, x}\right\|_{C} \leqq K_{7}\left\|p_{, x}\right\|_{H^{2}} ;\left\|q_{, x}\right\|_{L_{2}} \leqq\left\|q,{ }_{, x}\right\|_{H^{2}}$ and $\left\|\Delta\left(q,{ }_{, x}\right)\right\|_{L_{2}} \leqq\left\|q{ }_{, x}\right\|_{H^{2}}$ : these inequalities combine to give (i).

Proof of (ii).

$$
\begin{aligned}
\|(p \cdot \operatorname{grad}) q\|_{L_{2}} & \leqq\|p\|_{L_{2}}\left\|q, q_{, x}\right\|_{C} \leqq K_{7}\|p\|_{L_{2}}\left\|q q_{, x}\right\|_{H^{2}}, \\
\|\Delta((p \cdot \operatorname{grad}) q)\|_{L_{2}} & \leqq K_{7}\|\Delta p\|_{L_{2}}\left\|q q_{, x}\right\|_{H^{2}}+K\|p\|_{H^{2}}\left\|q,{ }_{, x}\right\|_{H^{2}}+K_{7}\|p\|_{H^{2}}\left\|q q_{, x}\right\|_{H^{2}} .
\end{aligned}
$$

These combine to give (ii). Note that $2 K_{7}<K_{5}$.

\section{Proof of (iii).}

$$
\begin{aligned}
\|(p \cdot \operatorname{grad}) q\|_{H^{1}}^{2}= & ((p \cdot \operatorname{grad}) q,(1-\Delta)((p \cdot \operatorname{grad}) q))_{L_{2}} \\
\leqq & \|p\|_{C}^{2}\left\|q,{ }_{, x}\right\|_{L_{2}}^{2}+\|p\|_{C}\left\|q_{, x}\right\|_{L_{2}}\left(K\|\Delta p\|_{H^{1}}\left\|q,{ }_{, x}\right\|_{H^{1}}+\left\|p{ }_{, x}\right\|_{C}\|q\|_{H^{2}}\right) \\
& +((p \cdot \operatorname{grad}) q,(p \cdot \operatorname{grad}) \Delta q)_{L_{2}} .
\end{aligned}
$$

Now, since $\nabla \cdot v=0$ and $p$ has the role of $v$ here, $\nabla \cdot p=0$ and

$$
\begin{aligned}
((p \cdot \operatorname{grad}) q,(p \cdot \operatorname{grad}) \Delta q)_{L_{2}} & =\left(p_{j} q_{i, x_{j}},\left(p_{k} \Delta q_{i}\right)_{, x_{k}}\right) \\
& =-\left(\left(p_{j} q_{i}, x_{j}\right), x_{k}, p_{k} \Delta q_{i}\right) \\
& \leqq K\|p\|\left\|_{C}^{2}\right\| q\left\|_{H^{2}}^{2}+K\right\| p,{ }_{, x}\left\|_{C}\right\| q_{, x}\left\|_{L_{2}}\right\| p\left\|_{C}\right\| q \|_{H^{2}} .
\end{aligned}
$$

The inequalities combine using Lemmas 0.1 and 0.2 to give (iii).

THEOREM 2.2. For any $[0, T]$ where $b$ and $v$ are defined, if $a \in H_{\sigma}^{3}, b \in C\left(T, H_{\sigma}^{2}\right)$ and $v \in C_{T}, \lim _{|x| \rightarrow \infty} v(x, t)=0$ uniformly in $t \in[0, T], v, x \in C\left(T, H^{2}\right), \nabla \cdot v=0$ then

$$
w(t)=e^{-t v A} a+\int_{0}^{t} e^{-(t-s) v A}(-(v \cdot \operatorname{grad}) w+(w \cdot \operatorname{grad}) v+\nu w+b) d s
$$

has a solution $w(t) \in C\left(T, H_{\sigma}^{2}\right)$ with $A^{1 / 2} w(t) \in C\left(T, H_{\sigma}^{2}\right)$ also and

$$
\lim _{t \downarrow 0}\left\|A^{1 / 2} w(t)-A^{1 / 2} a\right\|_{H_{\sigma}^{2}}=0 .
$$

Proof. The inequalities for $P_{v, v}$ and the continuity required by Theorem 2.1 follow from Lemma 2.2, the linearity of expressions like $(v \cdot \mathrm{grad}) w$ in both $v$ and $w$, and the properties assumed of $v$. Clearly $D\left(A^{1 / 2}\right) \subset D\left(P_{v, v}\right)$. Hence Theorem 2.1(I) gives the existence of solutions $w \in C\left(T, H_{\sigma}^{2}\right)$ of (2.2) with the proper requirements. 
III. For $u \in C\left(T, H_{\sigma}^{2}\right)$, we can form $F_{1}(u)=v$ satisfying the requirements of Theorem 1.1 and construct $w(t)$, the solution of Theorem 2.2 to

$$
w(t)=e^{-t v A} a+\int_{0}^{t} e^{-(t-s) v A}(-(v \cdot \operatorname{grad}) w+(w \cdot \operatorname{grad}) r+\nu w+b) d s
$$

which we call $F_{2}(v)=F_{2}\left(F_{1}(u)\right)$. We wish to establish that the mapping $F_{2}\left(F_{1}()\right): u \rightarrow w$ maps a closed convex set $S$ of a Banach space continuously into a relatively compact subset of $S$ and use Schauder's fixed point theorem to find a solution to (E)(a), (b), (c) and (d). The most important estimate giving $t$ independence of the viscosity $\nu$ comes from the following lemma.

Lemma 3.1. In any Hilbert space $H$, if $A$ is a positive selfadjoint operator, $q(t) \in C(T, H), a \in H$ and

$$
w(t)=e^{-t v A} a+\int_{0}^{t} e^{-(t-s) v A} q(s) d s
$$

then

$$
\|w(t)\|_{H}^{2} \leqq\|a\|_{H}^{2}+2 \int_{0}^{t}(q(s), w(s))_{H} d s .
$$

Proof. Suppose that $q(s)$ is Hölder-continuous in $s$. Then, from Lemma 2.1(vi), $d w / d t$ exists in $C([\varepsilon, T], H), \varepsilon>0 ; w \in D(A)(t>0)$ and $d w / d t+\nu A w=q(t)$ in $H$; $w(t) \rightarrow a$ strongly in $H$ as $t \rightarrow 0$. So

$$
(d w / d t, w)_{H}+\nu(A w, w)_{H}=(q(t), w(t))_{H} .
$$

Now $\nu(A w, w)_{H}=\nu\left\|A^{1 / 2} w\right\|_{H}^{2} \geqq 0$, so integrating the inequality gives

$$
\frac{1}{2}\left(\|w(t)\|_{H}^{2}-\|a\|_{H}^{2}\right)=\frac{1}{2} \int_{0}^{t} \frac{d}{d s}\|w\|_{H}^{2} d s \leqq \int_{0}^{t}(q(s), w(s))_{H} d s .
$$

By approximating strongly continuous $q(s)$ by Hölder-continuous functions, we can easily obtain the result for $q(s) \in C(T, H)$.

LeMmA 3.2. If $w \in C\left(T, H^{3}\right)$, then there is a constant $K_{8}$ such that

$$
\left|((v \cdot \operatorname{grad}) w, w)_{H^{2}}\right| \leqq K_{8}\|v, x\|_{H^{2}}\|w\|_{H^{2}}^{2} .
$$

Proof. If $w \in C_{0}^{\infty}$, then, since $\nabla \cdot v=0$,

$$
\begin{aligned}
((v \cdot \operatorname{grad}) w, w)_{H^{2}}= & ((1-\Delta)(v \cdot \operatorname{grad}) w,(1-\Delta) w)_{L_{2}} \\
= & ((v \cdot \operatorname{grad}) w, w)-((v \cdot \operatorname{grad}) w, \Delta w)-(\Delta(v \cdot \operatorname{grad}) w,(1-\Delta) w) \\
= & \frac{1}{2} \int \nabla \cdot\left(|w|^{2} v\right) d x-\left(\frac{\partial}{\partial x_{j}}\left(v_{j} w_{i}\right), \Delta w_{i}\right)-((\Delta v \cdot \operatorname{grad}) w,(1-\Delta) w) \\
& +\frac{1}{2} \int \nabla \cdot\left(|\Delta w|^{2} v\right) d x-\left(\frac{\partial}{\partial x_{j}}\left(v_{j} \Delta w_{i}\right), w_{i}\right) \\
& -2\left(\frac{\partial v_{j}}{\partial x_{K}} \frac{\partial^{2} w_{i}}{\partial x_{j} \partial x_{K}},(1-\Delta) w_{i}\right) .
\end{aligned}
$$


Now

$$
\frac{1}{2} \int \nabla \cdot\left(|w|^{2} v\right) d x=0=\frac{1}{2} \int \nabla \cdot\left(|\Delta w|^{2} v\right) d x
$$

and

$$
\left(\frac{\partial}{\partial x_{j}}\left(v_{j} w_{i}\right), \Delta w_{i}\right)=-\left(\frac{\partial}{\partial x_{j}}\left(v_{j} \Delta w_{i}\right), w_{i}\right)
$$

so four terms of the expression drop out, and we have

$$
\begin{aligned}
\left|((v \cdot \operatorname{grad}) w, w)_{H^{2}}\right| \leqq & \|(\Delta v \cdot \operatorname{grad}) w\|_{L_{2}}\|(1-\Delta) w\|_{L_{2}} \\
& +K\left\|\frac{\partial v_{j}}{\partial x_{K}} \frac{\partial^{2} w_{i}}{\partial x_{j} \partial x_{K}}\right\|_{L_{2}} \cdot\|(1-\Delta) w\|_{L_{2}} \\
\leqq & \left(K\|v, x\|_{H^{2}}\|w\|_{H^{2}}+K\|v, x\|_{C}\|w\|_{H^{2}}\right)\|w\|_{H^{2}}
\end{aligned}
$$

The result follows from a density argument for $w \in C\left(T, H^{3}\right)$ and the inequality $\|v, x\|_{C} \leqq K_{7}\|v, x\|_{H^{2}}$.

Lemma 3.3. Let $K_{9}=K_{5}+K_{8}$. Suppose that $a \in H_{\sigma}^{3}$ and $b \in C\left(T, H^{2}\right)$. Choose $T$ such that

$$
4 T\left(\nu+K_{9}\left(2\|a\|_{H^{2}}^{2}+K_{9}^{-1}\|b\|_{C\left(T, H^{2}\right)}\right)^{1 / 2}\right)<1
$$

and with $T$ so restricted, let

$$
M^{2}=2\|a\|_{H^{2}}^{2}+K_{9}^{-1}\|b\|_{C\left(T, H^{2}\right)} .
$$

Define $S=\left\{w \in C\left(T, H_{\sigma}^{2}\right) \mid\|w\|_{C\left(T, H^{2}\right)} \leqq M\right\}$. Then $F_{2}\left(F_{1}(S)\right) \subset S$.

Proof. By Theorem 1.1, if $u \in S$, then $F_{1}(u)$ exists. Since $u \in C\left(T, H^{2}\right), F_{1}(u) \in C_{T}$ and $\left(F_{1}(u)\right)_{, x} \in C\left(T, H^{2}\right)$ by inequalities (ii) and (iii) of Theorem 1.1. Lemma 0.3 and a study of Lemma 1.2 shows that $\lim _{|x| \rightarrow \infty} F_{1}(u)=0$ uniformly in $t$; hence we can apply Theorem 2.2 to obtain $w(t)=F_{2}\left(F_{1}(u)\right)$ satisfying (2.2) with $w \in C\left(T, H_{\sigma}^{3}\right)$. By Lemmas 2.2, 3.1 and 3.2

$$
\begin{aligned}
\|w(t)\|_{H^{2}}^{2} & \leqq\|a\|_{H^{2}}^{2}+2 \int_{0}^{t}(-(v \cdot \operatorname{grad}) w+(w \cdot \operatorname{grad}) v+\nu w+b, w)_{H^{2}} d s \\
& \leqq\|a\|_{H^{2}}^{2}+2 t\|w\|_{C\left(t, H^{2}\right)}^{2}\left(\left(K_{5}+K_{8}\right)\|v, x\|_{C\left(t, H^{2}\right)}+\nu\right)+2 t\|b\|_{C\left(t, H^{2}\right)}\|w\|_{C\left(t, H^{2}\right)} .
\end{aligned}
$$

Now $t \leqq T$ and $\|v, x\|_{C\left(t, H^{2}\right)}=\left\|F_{1}(u), x\right\|_{C\left(t, H^{2}\right)} \leqq\|u\|_{C\left(t, H^{2}\right)} \leqq M$; hence

$$
2 t\left(\left(K_{5}+K_{8}\right)\|v, x\|_{C\left(t, H^{2}\right)}+\nu\right) \leqq 2 T\left(K_{9} M+\nu\right)<\frac{1}{2}
$$

and we can rearrange the inequality to

$$
\begin{aligned}
\frac{1}{2}\|w\|_{C(t, H)}^{2} & \leqq\|a\|_{H^{2}}^{2}+2 t\|b\|_{C\left(t, H^{2}\right)}\|w\|_{C\left(t, H^{2}\right)} \\
& \leqq\|a\|_{H^{2}}^{2}+\left(2 K_{9} M\right)^{-1}\|b\|_{C\left(t, H^{2}\right)}\|w\|_{C\left(t, H^{2}\right)} .
\end{aligned}
$$

This quadratic expression in $\|w\|_{C\left(t, H^{2}\right)}$ can hold only if

$$
\begin{aligned}
\|w\|_{C\left(T, H^{2}\right)} & \leqq \frac{1}{2}\left(\|b\|_{C\left(T, H^{2}\right)}\left(K_{9} M\right)^{-1}+\left(\left(K_{9} M\right)^{-2}\|b\|_{C\left(T, H^{2}\right)}^{2}+8\|a\|_{H^{2}}^{2}\right)^{1 / 2}\right) \\
& =M \quad \text { which establishes that } F_{2}\left(F_{1}(S)\right) \subset S .
\end{aligned}
$$


Lemma 3.4. $S=\left\{w \in C\left(T, H_{\sigma}^{2}\right) \mid\|w\|_{C\left(T, H^{2}\right)} \leqq M\right\}$ is a closed convex set in $C\left(T, L_{2}\right)$.

Proof. Convexity is immediate. Suppose $w_{n} \rightarrow w$ in $C\left(T, L_{2}\right)$ as $n \rightarrow \infty$ with $w_{n} \in S$. Then $w \in C\left(T, L_{2}\right), F(w)$ exists and

$$
\left(\int_{B(0, N)}\left(1+|z|^{2}\right)^{2}|F(w)|^{2} d z\right)^{1 / 2} \leqq\left(1+N^{2}\right)\left\|F\left(w_{n}\right)-F(w)\right\|_{L_{2}}+\left\|F\left(w_{n}\right)\right\|_{H^{2}} \leqq \varepsilon+M
$$

for $n$ sufficiently large. This is true for any $N$; so $w \in C\left(T, H^{2}\right)$ and $\|w\|_{C\left(T, H^{2}\right)} \leqq M$. Also $(w, \nabla f)_{L_{2}}=\lim _{n \rightarrow \infty}\left(w_{n}, \nabla f\right)=0$ for smooth $f$ since $w_{n} \in S$. Hence $w \in S$.

Lemma 3.5. $F_{2}\left(F_{1}()\right): S \rightarrow S$ is continuous in the $C\left(T, L_{2}\right)$ topology.

Proof. Suppose $u_{n} \in S_{i}$ and $u_{n} \rightarrow u_{0} \in S$ in $C\left(T, L_{2}\right)$. Let $v_{i}=F_{1}\left(u_{i}\right), w_{i}=F_{2}\left(v_{i}\right)$ $=F_{2}\left(F_{1}\left(u_{i}\right)\right), i=0,1,2, \ldots$. We wish to use Lemma 3.1 with Hilbert space $L_{2}$; thus formally we must deal with the closure $A^{1}$ of $1-\Delta$ regarded as an operator on $L_{2}$. However, if $q \in H_{\sigma}^{2} \subset L_{2}$, then $A^{1} q=A q, e^{-t A^{1}} q=e^{-t A} q$ etc., so we can use our previous notation without difficulty. Now

$$
w_{i}(t)=e^{-t v A} a+\int_{0}^{t} e^{-(t-s) v A}\left(-\left(v_{i} \cdot \operatorname{grad}\right) w_{i}+\left(w_{i} \cdot \operatorname{grad}\right) v_{i}+\nu w_{i}+b\right) d s
$$

so by using Lemma 3.1 on the representation of $w_{i}-w_{0}$ and the fact that $\left(\left(v_{i} \cdot \operatorname{grad}\right)\left(w_{i}-w_{0}\right), w_{i}-w_{0}\right)_{L_{2}}=0$, we obtain

$$
\begin{aligned}
\left\|w_{i}(t)-w_{0}(t)\right\|_{L_{2}}^{2} \leqq 2 \int_{0}^{t}\left(-\left(\left(v_{i}-v_{0}\right)\right.\right. & \cdot \operatorname{grad}) w_{0}+\left(\left(w_{i}-w_{0}\right) \cdot \operatorname{grad}\right) v_{i} \\
& \left.\left.+\left(w_{0} \cdot \operatorname{grad}\right)\left(v_{i}-v_{0}\right), w_{i}-w_{0}\right)_{L_{2}}+\nu\left\|w_{i}-w_{0}\right\|_{L_{2}}^{2}\right) d s .
\end{aligned}
$$

The following inequalities hold by Lemmas $0.2,2.2$ and Theorem 1.1:

$$
\begin{aligned}
& \left\|\left(\left(v_{i}-v_{0}\right) \cdot \operatorname{grad}\right) w_{0}\right\|_{C\left(T, L_{2}\right)} \leqq K\left\|v_{i, x}-v_{0, x}\right\|_{C\left(T, L_{2}\right)}\left\|w_{0, x}\right\|_{C\left(T, H^{1}\right)}, \\
& \left\|\left(\left(w_{i}-w_{0}\right) \cdot \operatorname{grad}\right) v_{i}\right\|_{C\left(T, L_{2}\right)} \leqq\left\|v_{i, x}\right\|_{C_{T}} \cdot\left\|w_{i}-w_{0}\right\|_{C\left(T, L_{2}\right)}, \\
& \left\|\left(w_{0} \cdot \operatorname{grad}\right)\left(v_{i}-v_{0}\right)\right\|_{C\left(T, L_{2}\right)} \leqq\left\|w_{0}\right\|_{C_{T}} \cdot\left\|v_{i, x}-v_{0, x}\right\|_{C\left(T, L_{2}\right)}, \\
& \left\|v_{i, x}-v_{0, x}\right\|_{C\left(T, L_{2}\right)} \leqq\left\|u_{i}-u_{0}\right\|_{C\left(T, L_{2}\right)}, \\
& \left\|v_{i, x}\right\|_{C_{T}} \leqq K_{7}\left\|v_{i, x}\right\|_{C\left(T, H^{2}\right)} \leqq K_{7}\left\|u_{i}\right\|_{C\left(T, H^{2}\right)} \leqq K_{7} M \leqq K_{9} M .
\end{aligned}
$$

Hence using Schwarz' inequality

$$
\begin{aligned}
\left\|w_{i}-w_{0}\right\|_{C\left(T, L_{2}\right)}^{2} \leqq & 2 T\left(K_{9} M+\nu\right)\left\|w_{i}-w_{0}\right\|_{C\left(T, L_{2}\right)}^{2} \\
& +\left\|w_{i}-w_{0}\right\|_{C\left(T, L_{2}\right)} 2 T\left(K\left\|w_{0, x}\right\|_{C\left(T, H^{1}\right)}+\left\|w_{0}\right\|_{C_{T}}\right)\left\|u_{i}-u_{0}\right\|_{C\left(T, L_{2}\right)} .
\end{aligned}
$$

Since $4 T\left(K_{9} M+\nu\right)<1$, we easily obtain $\left\|w_{i}-w_{0}\right\|_{C\left(T, L_{2}\right)} \leqq K\left\|u_{i}-u_{0}\right\|_{C\left(T, L_{2}\right)}$ and the mapping is therefore continuous.

To show that $F_{2}\left(F_{1}(S)\right)$ is relatively compact in $C\left(T, L_{2}\right)$ we first show that it is an equicontinuous set of functions and then, with a somewhat intricate argument, show that the functions are uniformly small near infinity and thus we can use the Arzela-Ascoli theorem for compactness. 
LEMMA 3.6. For any $w \in F_{2}\left(F_{1}(S)\right)$,

(i) $\|w(t+h)-w(t)\|_{H^{2}} \leqq\left(h / v^{2}\right)^{1 / 4} K_{10}$,

(ii) $\|w(t+h)-w(t)\|_{H^{1}} \leqq h^{1 / 2} K_{11}$,

where $K_{10}$ and $K_{11}$ are independent of $\nu$ and depend only on $M$.

Proof. If $w \in F_{2}\left(F_{1}(S)\right)$, then, for some $u \in S, w=F_{2}\left(F_{1}(u)\right)=F_{2}(v)$ and

$$
\begin{gathered}
\begin{aligned}
& w(t)= e^{-t v A} a+\int_{0}^{t} e^{-(t-s) v A}(-(v \cdot \operatorname{grad}) w) d s+\int_{0}^{t} e^{-(t-s) v A}((w \cdot \operatorname{grad}) v+\nu w+b) d s \\
&= w^{1}(t)+w^{2}(t)+w^{3}(t) . \\
& w^{1}(t+h)-w^{1}(t)=\left(e^{-h v A}-1\right) A^{-1 / 2} e^{-t v A} A^{1 / 2} a, \text { so } \\
&\left\|w^{1}(t+h)-w^{1}(t)\right\|_{H^{1}} \leqq\left\|w^{1}(t+h)-w^{1}(t)\right\|_{H^{2}} \leqq K(h v)^{1 / 2}\left\|A^{1 / 2} a\right\|_{H^{2}}
\end{aligned}
\end{gathered}
$$

by Lemma 2.1(ix). Lemma 2.1(xi) applied to $w^{3}(t)$ (with $\delta=0$ ) gives

$$
\begin{aligned}
\left\|w^{3}(t+h)-w^{3}(t)\right\|_{H^{1}} & \leqq\left\|w^{3}(t+h)-w^{3}(t)\right\|_{H^{2}} \\
& \leqq K h^{1 / 2}\left(\|b\|_{C\left(T, H^{2}\right)}+\nu\|w\|_{C\left(T, H^{2}\right)}+\|w\|_{C\left(T, H^{2}\right)}\|v, x\|_{C_{T}}\right) \\
& \leqq K h^{1 / 2}\left(\|b\|_{C\left(T, H^{2}\right)}+\nu M+K_{3} M^{2}\right) .
\end{aligned}
$$

Now $A^{-1 / 2} w^{2}(t)=-\int_{0}^{t} e^{-(t-s) v A} A^{-1 / 2}(v \cdot \operatorname{grad}) w d s$, so by Lemma 2.1(xi) with $\delta=\frac{1}{2}$, $\mu=\frac{1}{4}$, we get

$$
\begin{aligned}
\left\|w^{2}(t+h)-w^{2}(t)\right\|_{H^{2}} & =\left\|A^{1 / 2}\left(A^{-1 / 2} w^{2}(t+h)-A^{-1 / 2} w^{2}(t)\right)\right\|_{H^{2}} \\
& \leqq h^{1 / 4} \nu^{-1 / 2} K\left\|A^{-1 / 2}(v \cdot \operatorname{grad}) w\right\|_{H^{2}} \\
& \equiv h^{1 / 4} \nu^{-1 / 2} K \cdot\|(v \cdot \operatorname{grad}) w\|_{H^{1}} \\
& \leqq h^{1 / 4} \nu^{-1 / 2} K \cdot K_{6} M^{2}\left(1+K_{3}\right) \quad \text { by Lemma 2.2(iii). }
\end{aligned}
$$

This establishes (i).

Lemma 2.1 will hold for $1-\Delta$ as an operator on $H^{1}$, and the result (ii) for $\left\|w^{2}(t+h)-w^{2}(t)\right\|_{H^{1}}$ independent of $v$ follows immediately from Lemma 2.1(xi) applied with $\delta=0$ to $H^{1}$.

LEMMA 3.7. $F_{2}\left(F_{1}(S)\right)$ is an equicontinuous set of functions for fixed $\nu>0$.

Proof. $S$ is uniformly Hölder-continuous in $x$ with exponent $\delta, \delta<\frac{1}{2}$ by Lemma 0.1. $F_{2}\left(F_{1}(S)\right)$ is uniformly Hölder-continuous in $t$ with exponent $\frac{1}{4}$ by Lemmas 0.1 and 3.6.

LEMMA 3.8. For any $\varepsilon>0$, there is an $N_{\varepsilon}$ such that if $w \in F_{2}\left(F_{1}(S)\right)$ then

$$
\sup _{t \in[0, T]} \int_{R^{3}-B\left(0, N_{\varepsilon}\right)}|w(t)|^{2} d x<\varepsilon^{2} .
$$

$N_{\varepsilon}$ is independent of $\nu>0$.

Proof. To obtain the result independent of $\nu$, we wish to use Lemma 3.1. To this end, we note that if $w(t)=e^{-t v A} a+\int_{0}^{t} e^{-(t-s) v A} q(s) d s$ with $q \in C\left(T, H^{2}\right)$ then, for any $C^{\infty}$ scalar function $f(x)$ bounded through its 4 th derivatives,

$$
w(t) f=e^{-t v A} a f+\int_{0}^{t} e^{-(t-s) v A}(q f-\nu w \Delta f-2 \nu(\nabla f \cdot \operatorname{grad}) w) d s .
$$


To establish this: if $q$ is Hölder-continuous, then $d w / d t+\nu A w=q$; hence, since $A=1-\Delta, d(w f) / d t+\nu A(w f)=q f-\nu w \Delta f-2 v(\nabla f \cdot \mathrm{grad}) w$ and the integral representation of $w f$ follows from semigroup theory. If $q$ is only in $C\left(T, H^{2}\right)$, we can approximate $q$ with Hölder-continuous functions and still obtain the integral equation representation for $w f$. Then let $f(r) \in C^{\infty}(0, \infty) ; 0 \leqq f(r) \leqq 1 ; f(r)=0$, $0 \leqq r \leqq 1 ; f(r)=1,2 \leqq r<\infty$; and use $f_{N}(x)=f(|x| / N)$ in the representation (3.1) with $q=(-(v \cdot \operatorname{grad}) w+(w \cdot \operatorname{grad}) v+\nu w+b)$ together with Lemma 3.1 applied $H=L_{2}$. Inequalities in Lemmas $0.2,2.2$, and Theorem 1.1, together with the restriction $4 T\left(K_{9} M+\nu\right)<1$ eventually yield an inequality of the form

$$
\left\|f_{N} w\right\|_{C\left(T, L_{2}\right)} \leqq K\left\|f_{N} a\right\|_{L_{2}}+K\left\|f_{N} b\right\|_{C\left(T, L_{2}\right)}+K / N
$$

where the third constant depends on $M$. Now

$$
\sup _{t \in[0, T]} \int_{R^{3}-B(0,2 N)}|w(t)|^{2} d x \leqq\left\|f_{N} w\right\|_{C\left(T, L_{2}\right)}^{2} \leqq \sup _{t \in[0, T]} \int_{R^{3}-B(0, N)}|w(t)|^{2} d x
$$

and similar inequalities for $\left\|f_{N} a\right\|_{L_{2}}$ and $\left\|f_{N} b\right\|_{C\left(T, L_{2}\right)}$ establish the result.

Lemma 3.9. $F_{2}\left(F_{1}(S)\right)$ is relatively compact in the topology $C\left(T, L_{2}\right)$ for fixed $\nu>0$.

Proof. Since, by Lemma 3.7, $F_{2}\left(F_{1}(S)\right)$ is equicontinuous, for any sequence $\left\{w_{i}\right\}$ by the Arzela-Ascoli theorem we can choose a subsequence that converges uniformly in $[0, T] \times B(0,1)$, a further subsequence that converges in $[0, T] \times B(0,2)$ and so forth; thus we can find a "diagonal" sequence $\left\{w_{j}\right\}$ that converges pointwise in $[0, T] \times R^{3}=Q_{T}$ and uniformly in any $[0, T] \times B(0, N)$ to some continuous $w$. Then

$$
\|w(t)\|_{L_{2}(B(0, N))} \leqq\left\|w(t)-w_{j}(t)\right\|_{L_{2}(B(0, N))}+\left\|w_{j}(t)\right\|_{L_{2}} \leqq \varepsilon+M
$$

if $j$ is large, for any $N$, gives $w(t) \in L_{2}$. Similar reasoning with Lemma 3.6 shows that $w(t) \in C\left(T, L_{2}\right)$. Finally

$$
\begin{aligned}
\left\|w_{i}(t)-w(t)\right\|_{L_{2}}^{2} & \leqq\left\|w_{i}(t)-w(t)\right\|_{L_{2}(B(0, N))}^{2}+2\left(\|w(t)\|_{L_{2}\left(R^{3}-B(0, N)\right)}^{2}+\left\|w_{i}(t)\right\|_{L_{2}\left(R^{3}-B(0, N)\right)}^{2}\right) \\
& \leqq \varepsilon
\end{aligned}
$$

for $N$ large and $i \geqq i_{0}$ also large, uniformly in $t \in[0, T]$, by Lemma 3.8, and this gives convergence in $C\left(T, L_{2}\right)$.

THEOREM 3.1. If $C \in C ; \lim _{|x| \rightarrow \infty} C(x)=0 ; \nabla \cdot C=0 ; a \equiv \nabla \times C \in H_{\sigma}^{3+\delta} ; B$ is $a$ continuous function on $Q_{T} ; B, x \in C\left(T, H^{2}\right) ; b \equiv \nabla \times B$ is continuous in tas a $C\left(T, H_{\sigma}^{2}\right)$ function and $T$ satisfies

$$
4 T\left(\nu+K_{9}\left(2\|a\|_{H^{2}}^{2}+K_{9}^{-1}\|b\|_{C\left(T, H^{2}\right)}\right)^{1 / 2}\right)<1,
$$

then there exist unique functions $w, v$, and $P(P$ is unique up to an arbitrary function of $t$ ) such that

(a) $\partial w / \partial t+(v \cdot \operatorname{grad}) w-(w \cdot \operatorname{grad}) v-\nu \Delta w=b$,

(b) $w(x, 0)=a(x)$,

(c) $\nabla \times v=w ; \nabla \cdot v=0$,

(d) $\lim _{|x| \rightarrow \infty} v(x, t)=0$ uniformly in $t \in[0, T]$, 
and

(a) $\partial v / \partial t+(v \cdot \operatorname{grad}) v-\nu \Delta v=-\operatorname{grad} P+B$,

(b) $v(x, 0)=C(x)$,

with $w \in C\left(T, H_{\sigma}^{3}\right) \cap C^{2+\mu}\left([\varepsilon, T] \times R^{3}\right) \cap C_{T}^{1+\mu} ; \partial w / \partial t \in C^{\mu}\left([\varepsilon, T] \times R^{3}\right)$ and $v \in C^{3+\mu}\left([\varepsilon, T] \times R^{3}\right) \cap C_{T}^{2+\mu} ; v{ }_{, x} \in C\left([\varepsilon, T], H^{4}\right) \cap C\left(T, H^{3}\right) ; \partial v / \partial t \in C^{1+\mu}\left(\left[\varepsilon, T \times R^{3}\right)\right.$ and $P, x \in C^{1+\mu}\left([\varepsilon, T] \times R^{3}\right)$ for any $\varepsilon>0$ and $0<\mu<\frac{1}{2}$.

$(\mathrm{E})(\mathrm{a})$ and $\left(\mathrm{E}^{1}\right)(\mathrm{a})$ are satisfied in the classical sense;

$$
\|v(t)-C\|_{C^{2+\mu}} ; \quad\|w(t)-a\|_{C^{1+\mu}} \text { and }\|w(t)-a\|_{H^{3}}
$$

all $\rightarrow 0$ as $t \rightarrow 0$.

Proof. First we prove the results for equations (E).

By the preceding lemmas of §III, Schauder's fixed point theorem can be applied to find some $w$ such that $w=F_{2}\left(F_{1}(w)\right)$. Let $v=F_{1}(w)$. Theorem 2.2 gives $w \in C\left(T, H_{\sigma}^{3}\right)$ and $\lim _{t \rightarrow 0}\|w(t)-a\|_{H^{3}}=0$. (E)(c) and (d) are satisfied by Theorem 1.1. To show that (a) is satisfied (Theorem 2.2 gives only the integral equation (2.2)) we must establish the necessary inequality for $P_{v, v}$ to use part (II) of Theorem 2.1. For this we must show that if $u \in H_{\sigma}^{3}=D\left(A^{1 / 2}\right)$ and $v=F_{1}(w)$, then there are some constants $K$ and $\mu>0$ such that

$$
\begin{aligned}
&\left\|\left(\left(v\left(t_{1}\right) \cdot \operatorname{grad}\right) u-(u \cdot \operatorname{grad}) v\left(t_{1}\right)\right)-\left(\left(v\left(t_{2}\right) \cdot \operatorname{grad}\right) u-(u \cdot \operatorname{grad}) v\left(t_{2}\right)\right)\right\|_{H^{2}} \\
& \leqq K\left|t_{1}-t_{2}\right|^{\mu}\left\|A^{1 / 2} u\right\|_{H^{2}} .
\end{aligned}
$$

First

$$
\left\|\left(\left(v\left(t_{1}\right)-v\left(t_{2}\right)\right) \cdot \operatorname{grad}\right) u\right\|_{H^{2}} \leqq K_{4}\left(\left\|v,{ }_{, x}\left(t_{1}\right)-v,{ }_{, x}\left(t_{2}\right)\right\|_{H^{2}}+\left\|v\left(t_{1}\right)-v\left(t_{2}\right)\right\|_{C}\right)\left\|A^{1 / 2} u\right\|_{H^{2}}
$$

and

$$
\left\|(u \cdot \operatorname{grad})\left(v\left(t_{1}\right)-v\left(t_{2}\right)\right)\right\|_{H^{2}} \leqq K_{5}\left\|v, x\left(t_{1}\right)-v,{ }_{, x}\left(t_{2}\right)\right\|_{H^{2}}\|u\|_{H^{2}}
$$

by Lemma 2.2. Theorem 1.1 gives

and

$$
\left\|v\left(t_{1}\right)-v\left(t_{2}\right)\right\|_{C}=\left\|F_{1}\left(w\left(t_{1}\right)-w\left(t_{2}\right)\right)\right\|_{C} \leqq K_{3}\left\|w\left(t_{1}\right)-w\left(t_{2}\right)\right\|_{H^{2}}
$$

$$
\left\|v,{ }_{, x}\left(t_{1}\right)-v,{ }_{, x}\left(t_{2}\right)\right\|_{H^{2}}=\left\|\left(F_{1}\left(w\left(t_{1}\right)-w\left(t_{2}\right)\right)\right),{ }_{, x}\right\|_{H^{2}} \leqq\left\|w\left(t_{1}\right)-w\left(t_{2}\right)\right\|_{H^{2}} .
$$

But $w$ is Hölder-continuous in $t$ by Lemma 3.6. Hence (a) is satisfied as in Theorem 2.1(II) and the smoothness of $v$ and $w$ is given by Lemma 0.1 applied with Theorems 1.1 and 2.1 ; thus the solution is classical.

For equations $\left(\mathrm{E}^{1}\right)$, we need to establish the differentiability of $v$ with respect to $t$. We have established that $\partial w / \partial t \in C\left([\varepsilon, T] ; H^{2}\right)$. Hence we can form $v^{1}(t)$ $\equiv F_{1}(\partial w / \partial t)$ for any $t>0$. Then, using the linearity of $F_{1}$ and Theorem 1.1, $v^{1}(t)$ $=\partial v / \partial t$ and $v^{1}(t)_{, x}=(\partial v / \partial t)_{, x} \in C\left([\varepsilon, T], H^{2}\right)$ by the following reasoning:

$$
\begin{aligned}
\|(v(t+\Delta t)-v(t)) / \Delta t-v(t)\|_{C} \\
=\left\|(1 / \Delta t) F_{1}(w(t+\Delta t)-w(t))-F_{1}(\partial w / \partial t)\right\|_{C} \\
\leqq K_{3}\|(1 / \Delta t)(w(t+\Delta t)-w(t))-\partial w / \partial t\|_{H^{2}} \rightarrow 0 \text { as } \Delta t \rightarrow 0 .
\end{aligned}
$$


Similarly $v^{1}(t), x=(\partial v / \partial t), x$. The smoothness of $v$ then follows from results for (E). Thus if $\partial v / \partial t+(v \cdot \operatorname{grad}) v-v \Delta v-B=u$, (E)(a) gives $\nabla \times u=0$ for $t>0$ and $u, x \in C\left([\varepsilon, T] ; H^{2}\right)$, which, by Lemma 0.1 gives $u \in C^{1+\mu}\left([\varepsilon, T] \times R^{3}\right)$. Thus there is a function $P$, unique to a function of $t$, such that $u=\nabla(-P)$ and

$$
P_{, x} \in C^{1+\mu}\left([\varepsilon, T] \times R^{3}\right)
$$

Now $C=F_{1}(a)$, so

and

$$
\|v(t)-C\|_{C}=\left\|F_{1}(w(t)-a)\right\|_{C} \leqq K_{3}\|w(t)-a\|_{H^{2}}
$$

$$
\left\|v,{ }_{, x}(t)-a, x\right\|_{C^{1+\mu}} \leqq K_{3, \mu}\|w(t)-a\|_{H^{3}}
$$

implies by the result for (E) that $\|v(t)-C\|_{C^{2+\mu}} \rightarrow 0$ as $t \rightarrow 0$.

We must establish uniqueness. Suppose that $\left(w^{1}, v^{1}\right)$ and $\left(w^{2}, v^{2}\right)$ are both solutions of $(E)$. Then

$$
w^{i}(t)=e^{-t v A} a+\int_{0}^{t} e^{-(t-s) v A}\left(-\left(v^{i} \cdot \operatorname{grad}\right) w^{i}+\left(w^{i} \cdot \operatorname{grad}\right) v^{i}+\nu w^{i}+b\right) d s .
$$

Applying Lemma 3.1 with $H=L_{2}$ to the integral representation of $w^{1}(t)-w^{2}(t)$ and recalling that $\left(\left(v^{1} \cdot \operatorname{grad}\right)\left(w^{1}-w^{2}\right), w^{1}-w^{2}\right)_{L_{2}}=0$, we obtain the inequality

$$
\begin{aligned}
\left\|w^{1}(t)-w^{2}(t)\right\|_{L_{2}}^{2} \leqq & 2 \int_{0}^{t}\left\|w^{1}(s)-w^{2}(s)\right\|_{L_{2}}\left\|\left(\left(v^{1}(s)-v^{2}(s)\right) \cdot \operatorname{grad}\right) w^{2}(s)\right\|_{L_{2}} d s \\
& +2 \int_{0}^{t}\left\|w^{1}-w^{2}\right\|_{L_{2}}\left(\left\|\left(w^{1} \cdot \operatorname{grad}\right)\left(v^{1}-v^{2}\right)\right\|_{L_{2}}\right. \\
& \left.+\left\|\left(\left(w^{1}-w^{2}\right) \cdot \operatorname{grad}\right) v^{2}\right\|_{L_{2}}+v\left\|w^{1}-w^{2}\right\|_{L_{2}}\right) d s .
\end{aligned}
$$

The uniqueness statement of Theorem 1.1 gives $v^{i}=F_{1}\left(w^{i}\right)$. Hence Lemma 0.2(iii) provides

$$
\left\|\left(\left(v^{1}-v^{2}\right) \cdot \operatorname{grad}\right) w^{2}\right\|_{L_{2}} \leqq K\left\|v^{1},{ }_{x}-v^{2}{ }_{, x}\right\|_{L_{2}}\left\|w^{2}\right\|_{H^{2}} \leqq K\left\|w^{1}-w^{2}\right\|_{L_{2}}\left\|w^{2}\right\|_{H^{2}} .
$$

Similar inequalities concerning the remaining terms yield an inequality of form $\left\|w^{1}(t)-w^{2}(t)\right\|_{L_{2}}^{2} \leqq K \int_{0}^{t}\left\|w^{1}(s)-w^{2}(s)\right\|^{2} d s$ which can only hold if $w^{1}=w^{2}$; hence $v^{1}=F\left(w^{1}\right)=F\left(w^{2}\right)=v^{2}$ also.

$\left(\mathrm{E}^{1}\right)$ will have a unique solution also, since if $(v, P)$ solves $\left(\mathrm{E}^{1}\right)$ then $(\nabla \times v, v)$ solves (E).

IV. In §III we established the existence, for any viscosity $\nu>0$, of a solution $\left(v^{v}, P^{v}\right)$ to the Navier-Stokes equations. In §IV we show that $v^{v}$ converges, as the viscosity $v$ goes to zero, to a function $v$ that gives the solution to the Euler equations for the flow of an ideal fluid in $R^{3}$.

The Euler equations are

$$
\begin{aligned}
& \partial v / \partial t+(v \cdot \operatorname{grad}) v=-\operatorname{grad} P+B \\
& \nabla \cdot v=0 \quad \text { with constraints } \\
& \lim _{|x| \rightarrow \infty} v(x, t)=0 \text { and } v(x, 0)=C(x) .
\end{aligned}
$$


By formally computing the curl of equation (4.1), we obtain the system

(i) $\partial v / \partial t+(v \cdot \operatorname{grad}) w-(w \cdot \operatorname{grad}) v=\nabla \times B \equiv b$,

(ii) $w(x, 0)=\nabla \times C(x) \equiv a(x)$,

(iii) $\nabla \times v=w ; \nabla \cdot v=0$,

(iv) $\lim _{|x| \rightarrow \infty} v(x, t)=0$.

Assume for this section that $0<\nu \leqq \nu_{0}, T$ satisfies

$$
4 T\left(\nu_{0}+K_{9}\left(2\|a\|_{H^{2}}^{2}+K_{9}^{-1}\|b\|_{C\left(T, H^{2}\right)}\right)^{1 / 2}\right)<1
$$

and $M^{2}=2\|a\|_{H^{2}}^{2}+K_{9}^{-1}\|b\|_{C\left(T, H^{2}\right)}$ where $a \equiv \nabla \times C$ and $b \equiv \nabla \times B$. Use these restrictions and functions to obtain the results of §III for various viscosities $\nu$.

LEMMA 4.1. If $\left(w^{v}, v^{v}\right)$ is the solution of (E) with viscosity $\nu \leqq \nu_{0}$ of Theorem 3.1, then there is a function $w \in C\left(T, H_{\sigma}^{2}\right) ;\|w\|_{C\left(T, H^{2}\right)} \leqq M$ such that if $v=F_{1}(w)$, then

(i) $\left\|w^{v}-w\right\|_{C\left(T, L_{2}\right)} \leqq 8 T M \nu$,

(ii) $\left\|v^{v}-v\right\|_{C\left(T, L_{6}\right)} \leqq 8 T M K_{2} \nu$,

(iii) $\left\|v^{v}, x-v, x\right\|_{C\left(T, L_{2}\right)} \leqq 8 T M \nu$.

Proof. Let $\left(w^{i}, v^{i}\right)$ be solutions of (E) with viscosities $\nu_{i}$ of Theorem 3.1. Using the notation $A=1-\Delta$, Theorem 3.1 gives

$$
\begin{aligned}
& d\left(w^{i}-w^{j}\right) / d t+\nu_{i} A\left(w^{i}-w^{j}\right)=p \\
& \equiv\left(v_{j}-v_{i}\right)\left(A w^{j}-w^{i}\right)+v_{j}\left(w^{i}-w^{j}\right)+\left(\left(w^{i}-w^{j}\right) \cdot \operatorname{grad}\right) v^{i} \\
& \quad+\left(w^{j} \cdot \operatorname{grad}\right)\left(v^{i}-v^{j}\right)+\left(\left(v^{j}-v^{i}\right) \cdot \operatorname{grad}\right) w^{i}+\left(v^{j} \cdot \operatorname{grad}\right)\left(w^{j}-w^{i}\right) .
\end{aligned}
$$

The initial value of $w^{i}-w^{j}=a-a=0$, so we can use Lemma 3.1 with Hilbert space $L_{2}$ and the result $\left(\left(v^{j} \cdot \operatorname{grad}\right)\left(w^{j}-w^{i}\right), w^{j}-w^{i}\right)_{L_{2}}=0$ to obtain.

$$
\begin{aligned}
\left\|w^{i}-w^{j}\right\|_{C\left(t, L_{2}\right)} \leqq & 2 t\left|v_{j}-v_{i}\right|\left(\left\|A w^{j}\right\|_{C\left(t, L_{2}\right)}+\left\|w^{i}\right\|_{C\left(t, L_{2}\right)}\right)+2 t\left(v_{j}+\left\|v^{i},{ }_{, x}\right\|_{C_{t}}\right)\left\|w^{i}-w^{j}\right\|_{C\left(t, L_{2}\right)} \\
& +2 t\left(\left\|w^{j}\right\|_{C_{t}} \cdot\left\|v^{i}, x-v^{j}{ }_{, x}\right\|_{C\left(t, L_{2}\right)}+\left\|\left(\left(v^{j}-v^{i}\right) \cdot \operatorname{grad}\right) w^{i}\right\|_{C\left(t, L_{2}\right)}\right) .
\end{aligned}
$$

Now (see proof of Lemma 3.2)

and

$$
\left\|\left(\left(v^{j}-v^{i}\right) \cdot \operatorname{grad}\right) w^{i}\right\|_{C\left(t, L_{2}\right)} \leqq K_{8}\left\|v_{, x}^{j}-v^{i}, x\right\|_{C\left(t, L_{2}\right)}\left\|w^{i},{ }_{, x}\right\|_{C\left(t, H^{1}\right)}
$$

So

$$
\left\|v^{i}, x-v^{j}, x\right\|_{C\left(t, L_{2}\right)}=\left\|F_{1}\left(w^{i}-w^{j}\right), x\right\|_{C\left(t, L_{2}\right)} \leqq\left\|w^{i}-w^{j}\right\|_{C\left(t, L_{2}\right)} .
$$

$$
\left\|w^{i}-w^{j}\right\|_{C\left(t, L_{2}\right)} \leqq 4 t M\left|\nu_{j}-\nu_{i}\right|+2 t\left(\nu_{j}+\left(2 K_{7}+K_{8}\right) M\right)\left\|w^{i}-w^{j}\right\|_{C\left(t, L_{2}\right)},
$$

since both $w^{i}$ and $w^{j}$ are bounded in $C\left(t, H^{2}\right)$ by $M$. Then

$$
\left\|w^{i}-w^{j}\right\|_{C\left(T, L_{2}\right)} \leqq 8 T M\left|\nu_{i}-v_{j}\right|
$$

follows from $2 t\left(\nu_{j}+\left(2 K_{7}+K_{8}\right) M\right) \leqq 2 T\left(\nu_{0}+K_{9} M\right)<\frac{1}{2}$. Both $w^{i}$ and $w^{j} \in S$ where

$$
S=\left\{w \in C\left(T, H_{\sigma}^{2}\right) \mid\|w\|_{C\left(T, H^{2}\right)} \leqq M\right\}
$$


which is closed in $C\left(T, L_{2}\right)$ by Lemma 3.4; hence $w \in S$ and $v=F_{1}(w)$ exist and the inequalities follow from Theorem 1.1 and (4.5).

THEOREM 4.1. If $C \in C, \lim _{|x| \rightarrow \infty} C(x)=0, \nabla \cdot C=0, a \equiv \nabla \times C \in H^{3+\delta}$ and $B$ is $a$ continuous function such that $B, x \in C\left(T, H^{2}\right)$ and $b \equiv \nabla \times B$ is Hölder-continuous in $t$ as a $C\left(T, H^{2}\right)$ function, $T$ satisfies

$$
4 T\left(\nu_{0}+K_{9}\left(2\|\nabla \times C\|_{H^{2}}^{2}+K_{9}^{-1}\|\nabla \times B\|_{C\left(T, H^{2}\right)}\right)^{1 / 2}\right)<1
$$

then there are unique functions $w, v$ and $P$ ( $P$ is unique up to an arbitrary function of $t)$ in $[0, T]$ such that

(i) $\partial w / \partial t+(v \cdot \operatorname{grad}) w-(w \cdot \operatorname{grad}) v=b$

(ii) $w(x, 0)=a(x)$,

(iii) $\nabla \times v=w ; \nabla \cdot v=0$,

(iv) $\lim _{|x| \rightarrow \infty} v(x, t)=0$ uniformly in $t \in[0, T]$,

and

(i) $\partial v / \partial t+(v \cdot \operatorname{grad}) v=-\operatorname{grad} P+B$,

(ii) $v(x, 0)=C(x)$,

with $\quad w \in C\left(T, H^{2}\right) ; \quad \partial w / \partial t \in C\left(T, H^{1}\right) ; \quad v \in C_{T}^{1+\mu} ; \quad v, x \in C\left(T, H^{2}\right) ; \quad \partial v / \partial t \in C^{\mu}$ (locally in $x \in R^{3}$ uniformly in $\left.t \in[0, T]\right) ; \partial v / \partial t \in C\left(T, L_{6}\right) ; \partial v, x / \partial t \in C\left(T, H^{1}\right)$; $\partial(\operatorname{grad} P) / \partial t \in C\left(T, H^{1}\right)$ for $\mu<\frac{1}{2}$.

$\|w(t)-a\|_{H^{1}} ;\|v(t)-C\|_{C} ;\|v(t)-C\|_{L_{6}}$ and $\left\|v{ }_{, x}(t)-C{ }_{, x}\right\|_{H^{1}}$ all go to zero as $t \rightarrow 0$.

If $\left(v^{v}, P^{v}\right)$ is the solution to the Navier-Stokes flow of Theorem 3.1 with $\nu<\nu_{0}$, then $\lim _{v \rightarrow 0}\left\|v^{v}-v\right\|_{C_{T}}=0 ;\left\|v^{v}-v\right\|_{C\left(T, L_{6}\right)} \leqq 8 T M K_{4} \nu$ and $\left\|v^{v}, x-v{ }_{, x}\right\|_{C\left(T, L_{2}\right)} \leqq 8 T M v$.

Proof. We first show that $(w, v)$ of Lemma 4.1 is a "weak solution" of (4.2)(i). Now

$$
\begin{aligned}
&\left\|\left(w^{v} \cdot \operatorname{grad}\right) v^{v}-(w \cdot \operatorname{grad}) v\right\|_{C\left(T, L_{2}\right)} \\
& \leqq\left\|\left(\left(w^{v}-w\right) \cdot \operatorname{grad}\right) v^{v}\right\|_{C\left(T, L_{2}\right)}+\left\|(w \cdot \operatorname{grad})\left(v^{v}-v\right)\right\|_{C\left(T, L_{2}\right)} \\
& \leqq\left\|w^{v}-w\right\|_{C\left(T, L_{2}\right)}\left\|v^{v}, x\right\|_{C_{T}}+\|w\|_{C_{T}}\left\|v^{v}, x-v, x\right\|_{C\left(T, L_{2}\right)} \\
& \rightarrow 0 \text { as } \nu \rightarrow 0
\end{aligned}
$$

by Lemma 4.1 and the uniform (in $\nu$ ) boundedness of $\left\|v^{v},{ }_{, x}\right\|_{C_{T}}\left(w^{v} \in S\right.$ for all $\nu>0)$. For $p(x) \in C_{0}^{\infty}$,

$$
\begin{gathered}
\left(\left(v^{v} \cdot \operatorname{grad}\right) w^{v}-(v \cdot \operatorname{grad}) w, p\right)_{L_{2}}=\left(\left(\left(v^{v}-v\right) \cdot \operatorname{grad}\right) w^{v}, p\right)_{L_{2}}+\left((v \cdot \operatorname{grad})\left(w^{v}-w\right), p\right)_{L_{2}} . \\
\left\|\left(\left(v^{v}-v\right) \cdot \operatorname{grad}\right) w^{v}\right\|_{L_{2}} \leqq K\left\|v^{v}, x-v, x\right\|_{L_{2}}\left\|w^{v}\right\|_{H^{2}} \rightarrow 0 \quad \text { as } \nu \rightarrow 0
\end{gathered}
$$

uniformly in $t$ by Lemmas 0.2 (iii) and 4.1.

$$
\begin{aligned}
\left|\left((v \cdot \operatorname{grad})\left(w^{v}-w\right), p\right)_{L_{2}}\right| & =\left|\left(\partial\left(v_{j}\left(w^{v}-w\right)\right) / \partial x_{j}, p\right)_{L_{2}}\right| \\
& =\left|\left(v_{j}\left(w^{v}-w\right), \partial p / \partial x_{j}\right)_{L_{2}}\right| \\
& \leqq K\|v\|_{C_{T}} \cdot\left\|w^{v}-w\right\|_{L_{2}}\left\|p_{, x}\right\|_{L_{2}} \rightarrow 0 \quad \text { uniformly in } t \in[0, T]
\end{aligned}
$$


Hence

$$
\left(\left(v^{v} \cdot \operatorname{grad}\right) w^{v}, p\right)_{L_{2}} \rightarrow((v \cdot \operatorname{grad}) w, p)_{L_{2}} \quad \text { as } \nu \rightarrow 0 .
$$

From Theorem 3.1, $\partial w^{v} / \partial t=b+\left(w^{v} \cdot \operatorname{grad}\right) v^{v}-\left(v^{v} \cdot \operatorname{grad}\right) w^{v}+\nu \Delta w^{v}$ so, for $p \in C_{0}^{\infty}$,

$$
\begin{gathered}
\int_{0}^{t}\left(b+\left(w^{v} \cdot \operatorname{grad}\right) v^{v}-\left(v^{v} \cdot \operatorname{grad}\right) w^{v}, p\right)_{L_{2}} d s+v \int_{0}^{t}\left(\Delta w^{v}, p\right)_{L_{2}} d s \\
=\int_{0}^{t} \frac{d}{d s}\left(w^{v}, p\right)_{L_{2}} d s=\left(w^{v}(t)-a, p\right)_{L_{2}} .
\end{gathered}
$$

Inequalities (4.6), (4.7), $\left|\left(\Delta w^{v}, p\right)_{L_{2}}\right| \leqq\left\|w^{v}\right\|_{H^{2}}\|p\|_{C}$, and Lemma 4.1 then give

$$
(w(t)-a, p)_{L_{2}}=\int_{0}^{t}(b+(w \cdot \operatorname{grad}) v-(v \cdot \operatorname{grad}) w, p)_{L_{2}} d s .
$$

Let $q(t) \equiv b+(w \cdot \operatorname{grad}) v-(v \cdot \operatorname{grad}) w \in C\left(T, H^{1}\right)$. If $u(t)=a+\int_{0}^{t} q(s) d s$, then $u(t)$ and $d u / d t=q(t) \in C\left(T, H^{1}\right)$ and $\|u(t)-a\|_{H^{1}} \rightarrow 0$. Hence

$$
(u(t)-a, p)_{L_{2}}=\int_{0}^{t}(q, p)_{L_{2}} d s=(w(t)-a, p)_{L_{2}},
$$

which can only occur if $u=w$, which establishes (4.2)(i) and (ii). (4.2)(iii) is true since $v=F_{1}(w) . \lim _{|x| \rightarrow \infty} v(x, t)=0$ uniformly in $t \in[0, T]$ follows from Lemmas $0.3,3.8$ and a study of the proof of Lemma 1.2(i).

To show differentiability in $t$ of $v$, let $q_{h}(t)=(q(t+h)-q(t)) / h$ for any function $q$. Then by the results of Theorem 1.1 and the linearity of $F_{1}$,

$$
\left\|(v, x)_{h_{1}}(t)-(v, x)_{h_{2}}(t)\right\|_{H^{1}} \leqq\left\|w_{h_{1}}(t)-w_{h_{2}}(t)\right\|_{H^{1}} .
$$

Since $\partial w / \partial t$ exists in $C\left(T, H^{1}\right), \partial(v, x) / \partial t$ will exist in $H^{1}$ for any $t \in[0, T]:$ strong continuity in $t$ follows from a similar inequality and the strong continuity of $\partial w / \partial t$. Parallel reasoning and inequality (i) of Theorem 1.1 shows that $\partial v / \partial t$ exists in $C\left(T, L_{6}\right)$. Lemma 0.2 (iv) then gives $\partial v / \partial t \in C^{\lambda}([0, T] \times B(0, N)), \lambda<\frac{1}{2}$, for any $N$. Thus we can write (4.2)(i) as $\nabla \times q=0$, where $q=\partial v / \partial t+(v \cdot \operatorname{grad}) v-B \in C^{\lambda}$ (locally). Define $P(x, t)=-\int_{\Gamma} q(x, t) d \sigma$ where $\Gamma$ is any smooth path from 0 to $x$. If $q$ is sufficiently smooth, the condition $\nabla \times q=0$ guarantees that $P(x, t)$ is defined independent of choice of path $\Gamma$; by use of mollifier theory we can construct smooth approximations to $q \in C^{\lambda}$ (locally) preserving the property $\nabla \times q=0$ and easily obtain this result for $q$ only in $C^{\lambda}$ (locally). Then

$$
\partial v / \partial t+(v \cdot \operatorname{grad}) v=-\operatorname{grad} P+B
$$

and the statements concerning the smoothness of $v$ follow from the results for system (4.2).

The properties postulated for $C$ give $C=F_{1}(\nabla \times C)$, so

$$
\|v(t)-C\|_{L_{6}}=\left\|F_{1}(w)(t)-F_{1}(\nabla \times C)\right\|_{L_{6}} \leqq K_{2}\|w(t)-\nabla \times C\|_{L_{2}} \rightarrow 0 \quad \text { as } t \rightarrow 0 .
$$

Similar reasoning and Theorem 1.1 gives

$$
\|v, x(t)-C, x\|_{H^{1}} \leqq\|w(t)-\nabla \times C\|_{H^{1}} \rightarrow 0 \text { as } t \rightarrow 0 .
$$


Lemma 0.7 (iv) then gives $\|v(t)-C\|_{C(B(0, N))} \rightarrow 0$ as $t \rightarrow 0 ; v(t)$ is uniformly (in $t$ ) small near $\infty$; $C$ is small near $\infty$; hence $\|v(t)-C\|_{C} \rightarrow 0$ as $t \rightarrow 0$.

To show uniqueness: If $\left(w^{1}, v^{1}\right)$ and $\left(w^{2}, v^{2}\right)$ are both solutions of (4.2), then $v^{i}=F\left(w^{i}\right)$ by the uniqueness statement of Theorem 1.1. Also

$$
\begin{aligned}
&\left(d\left(w^{1}-w^{2}\right) / d t, w^{1}-w^{2}\right)_{L_{2}} \\
&=\left(\left(\left(w^{1}-w^{2}\right) \cdot \operatorname{grad}\right) v^{1}, w^{1}-w^{2}\right)_{L_{2}}+\left(\left(w^{2} \cdot \operatorname{grad}\right)\left(v^{1}-v^{2}\right)+\left(\left(v^{2}-v^{1}\right) \cdot \operatorname{grad}\right) w^{1}\right. \\
&\left.+\left(v^{2} \cdot \operatorname{grad}\right)\left(w^{2}-w^{1}\right), w^{1}-w^{2}\right)_{L_{2}} .
\end{aligned}
$$

Since $w^{1}(0)-w^{2}(0)=a-a=0$ and $\left(v^{2} \cdot \operatorname{grad}\left(w^{2}-w^{1}\right), w^{1}-w^{2}\right)_{L_{2}}=0$ we can use estimates similar to those of the uniqueness proof of Theorem 3.1 to obtain an inequality of form $\left\|w^{1}(t)-w^{2}(t)\right\|^{2} \leqq K \int_{0}^{t}\left\|w^{1}(s)-w^{2}(s)\right\|^{2} d s$, which can only occur if $w^{1}=w^{2}$; hence $v^{1}=F_{1}\left(w^{1}\right)=F_{1}\left(w^{2}\right)=v^{2}$ also. $(v, P)$ is a unique solution of (4.1) since $(\nabla \times v, v)$ is a unique solution of (4.2).

Lemma 4.1 gives the convergence of $v^{v}$ to $v$ except for the result $\left\|v^{v}-v\right\|_{C_{T}} \rightarrow 0$ as $\nu \rightarrow 0$. We prove this by contradiction. Suppose, for some $\varepsilon>0$, there is a sequence of $\nu_{i}$ with associated $v_{i} \equiv v^{v_{i}}$ such that $\left\|v_{i}-v\right\|_{C_{T}}>\varepsilon$. Let $w_{i}=F_{2}\left(v_{i}\right)$; $w_{i} \in F_{2}\left(F_{1}(S)\right)$, hence Lemma 3.8, Lemma 0.3 and Lemma 1.2 show that $v_{i}$ is small near $\infty$ uniformly in $t$ and independent of $\nu$. Hence $\left\|v_{i}-v\right\|_{C_{T}}>\varepsilon$ occurs only within some ball $B(0, N)$; i.e., $\left\|v_{i}-v\right\|_{C([0, T] \times B(0, N))}>\varepsilon$. Lemma $0.1(\mathrm{iv}),\left\|v_{i}\right\|_{C\left(T, L_{6}\right)}$ $\leqq K_{2}\left\|w_{i}\right\|_{C\left(T, L_{2}\right)} \leqq K_{2} M$ and $\left\|\left(v_{i}\right), x\right\|_{C\left(T, H^{1}\right)} \leqq B$ show that $\left\{v_{i}\right\}$ are equicontinuous in $x \in B(0, N)$. The same inequality can be used with Lemma 3.6(ii) to establish that $\left\{v_{i}\right\}$ are equicontinuous in $t$ (independent of $\nu$ ). Hence there exists, by the ArzelaAscoli theorem, a subsequence $\left\{v_{j}\right\}$ and $v^{\prime} \in C([0, T] \times B(0, N))$ such that $\left\|v_{j}-v^{\prime}\right\|_{C([0, T] \times B(0, N))} \rightarrow 0$ as $j \rightarrow \infty$; hence $\left\|v_{j}-v^{\prime}\right\|_{C\left([0, T], L_{6}(B(0, N))\right)} \rightarrow 0$ as $j \rightarrow \infty$ also. But $v_{j} \rightarrow v$ in $C\left(T, L_{6}\right)$, which implies that $v=v^{\prime}$ and contradicts the assumption that $\left\|v_{i}-v\right\|_{C([0, T] \times B(0, N))}>\varepsilon$. Thus $\left\|v^{\nu}-v\right\|_{C_{T}} \rightarrow 0$ as $\nu \rightarrow 0$.

\section{BIBLIOGRAPHY}

1. O. A. Ladyženskaja, Mathematical problems in the dynamics of a viscous incompressible fluid, Fizmatgiz, Moscow, 1961; English transl., Gordon and Breach, New York, 1963. MR 27 \#5034a,b.

2. R. Courant, Methods of mathematical physics. Vol. II: Partial differential equations, Interscience, New York, 1962. MR 25 \#4216.

3. T. Kato and H. Fujita, On the nonstationary Navier-Stokes system, Rend. Sem. Mat. Univ. Padova 32 (1962), 243-260. MR 26 \#495.

4. H. Fujita and T. Kato, On the Navier-Stokes initial value problem. I, Arch. Rational Mech. Anal. 16 (1964), 269-315. MR 29 \#3774.

5. K. K. Golovkin, Vanishing viscosity in Cauchy's problem for hydromechanics, Trudy Mat. Inst. Steklov. 92 (1966), 31-49= Proc. Steklov Inst. Math. 92 (1966), 33-53. MR 34 \#7097.

6. E. Hopf, Über die Anfangswertaufgabe für die hydrodynamischen Grundgleichungen, Math. Nachr. 4 (1951), 213-231. MR 14, 327.

7. V. I. Judovič, Non-stationary flows of an ideal incompressible fluid, Ž. Vyčisl. Mat. i Mat. Fiz. 3 (1963), 1032-1066. MR 28 \#1415.

8. J. Leray, Sur le mouvement d'un liquide visqueux emplissant l'espace, Acta Math. 63 (1934), 193-248. 
9. L. Lichtenstein, Grundlagen der Hydromechanik, Springer-Verlag, Berlin, 1929.

10. F. J. McGrath, Nonstationary plane flow of viscous and ideal fluids, Arch. Rational Mech. Anal. 27 (1968), 329-348. MR 36 \#4870.

11. C. W. Oseen, Neuere Methoden und Ergebnisse in der Hydrodynamik, Akademie Verlag, Leipzig, 1927.

12. K. Yosida, Functional analysis, Die Grundlehren der math. Wissenschaften, Band 123, Academic Press, New York; Springer-Verlag, Berlin, 1965. MR 31 \#5054.

13. T. Kato, Perturbation theory for linear operators, Die Grundlehren der math. Wissenschaften, Band 132, Springer-Verlag, New York, 1966, chap. 9. MR 34 \#3324.

14. L. Bers, F. John and M. Schechter, Partial differential equations, Lectures in Appl. Math., vol. 3, Interscience, New York, 1964. MR 29 \#346.

15. D. G. Ebin and J. E. Marsden, Groups of diffeomorphisms and the solution of the classical Euler equations for a perfect fluid, Bull. Amer. Math. Soc. 75 (1969), 962-967. MR 39 \#7632.

16. J. E. Marsden, Non-linear semi-groups associated with the equations for a non-homogeneous fluid, University of California, Berkeley, Calif., 1970 (unpublished).

San Jose State College,

San Jose, California 95114 